\title{
Deficiency of Socs3 leads to brain- targeted experimental autoimmune encephalomyelitis via enhanced neutrophil activation and ROS production
}

\author{
Zhaoqi Yan, ${ }^{1}$ Wei Yang, ${ }^{1}$ Luke Parkitny, ${ }^{1}$ Sara A. Gibson, ${ }^{2}$ Kevin S. Lee, ${ }^{1}$ Forrest Collins, ${ }^{1}$ \\ Jessy S. Deshane, ${ }^{3}$ Wayne Cheng, ${ }^{4}$ Amy S. Weinmann, ${ }^{4}$ Hairong Wei, ${ }^{1}$ Hongwei Qin, ${ }^{1}$ \\ and Etty N. Benveniste ${ }^{1}$ \\ 'Department of Cell, Developmental and Integrative Biology, University of Alabama at Birmingham, Birmingham, \\ Alabama, USA. 'Lineberger Comprehensive Cancer Center, University of North Carolina at Chapel Hill, Chapel Hill, North \\ Carolina, USA. ${ }^{3}$ Department of Medicine, and ${ }^{4}$ Department of Microbiology, University of Alabama at Birmingham, \\ Birmingham, Alabama, USA.
}

\begin{abstract}
Dysregulation of the JAK/STAT signaling pathway is associated with multiple sclerosis (MS) and its mouse model, experimental autoimmune encephalomyelitis (EAE). Suppressors of cytokine signaling (SOCS) negatively regulate the JAK/STAT pathway. We previously reported a severe, brain-targeted, atypical form of EAE in mice lacking Socs3 in myeloid cells (Socs $3^{\mathrm{LL} y \mathrm{MM}}$ ), and that this atypical EAE is associated with cerebellar neutrophil infiltration. There is emerging evidence that neutrophils are detrimental in the pathology of MS/EAE; however, their exact function is unclear. Here we demonstrate that neutrophils from the cerebellum of Socs $3^{\mathrm{ALysM}}$ mice show a hyperactivated phenotype with excessive production of reactive oxygen species (ROS) at the peak of EAE. Neutralization of ROS in vivo delayed the onset and reduced severity of atypical EAE. Mechanistically, Socs3-deficient neutrophils exhibited enhanced signal transducer and activator of transcription 3 (STAT3) activation, a hyperactivated phenotype in response to granulocyte colonystimulating factor (G-CSF), and upon G-CSF priming, increased ROS production. Neutralization of G-CSF in vivo significantly reduced the incidence and severity of the atypical EAE phenotype. Overall, our work elucidates that hypersensitivity of G-CSF/STAT3 signaling in Socs $3^{\text {LLysM }}$ mice leads to atypical EAE by enhanced neutrophil activation and increased oxidative stress, which may explain the detrimental role of G-CSF in MS patients.
\end{abstract}

Conflict of interest: The authors have declared that no conflict of interest exists.

Copyright: (c) 2019 American Society for Clinical Investigation

Submitted: November 30, 2018 Accepted: March 27, 2019 Published: May 2, 2019.

Reference information: /CI Insight. 2019;4(9):e126520. https://doi. org/10.1172/jici.nsight.126520

\section{Introduction}

Multiple sclerosis (MS) is an autoimmune disease that affects the CNS (1). The disease causes severe demyelination and neuronal loss in both the brain and spinal cord, which leads to neurological dysfunction, including visual loss, limb weakness, ataxia, and impaired cognition $(2,3)$. Although there are 17 FDA-approved disease-modifying therapies, none is able to stop disease progression (3). The pathobiology of MS is very complex, and both innate and adaptive immunity contribute to disease development and progression (4). Experimental autoimmune encephalomyelitis (EAE) is a widely used mouse model of MS mediated by autoimmune $\mathrm{CD}^{+} \mathrm{T}$ cells and cells of the innate immune system (5). Clinical and histopathological similarities between MS and EAE allow findings obtained from the EAE model to be extrapolated to patients with MS.

Neutrophils are the most abundant cell type in human blood and are the first responders of the immune system when the body encounters foreign antigens or sterile inflammation $(6,7)$. Neutrophils initiate immune responses by secretion of proinflammatory cytokines/chemokines, degranulation, generation of ROS, formation of neutrophil extracellular traps (NETs), and antigen presentation $(6,8-10)$. Neutrophils are involved in the pathology of CNS autoimmune diseases, such as MS/EAE and neuromyelitis optica spectrum disorder (NMOSD) (11-15). Neutrophils contribute to EAE disease by secretion of proinflammatory cytokines $(16,17)$, promoting maturation of antigen-presenting cells, such as dendritic cells and macrophages (18), and disruption of the blood-spinal cord barrier (19). Neutrophil-related chemokines/mediators (G-CSF, CXCL1, CXCL8, 
CXCL5, and neutrophil elastase) correlate with neurological disability and lesion burden in MS patients (20). Neutrophils from MS patients exhibit a primed, hyperactivated phenotype compared with healthy controls (21). Moreover, several studies indicate that administration of granulocyte colony-stimulating factor (G-CSF), the cytokine that is critical for maturation of neutrophils, leads to MS relapses (22, 23). NMOSD is an autoimmune demyelinating disease distinct from MS, characterized by optic neuritis, astrocytopathy and serum antibodies against Aquaporin-4 (24). Neutrophils are prominent in CNS lesions and cerebrospinal fluid from NMOSD patients (25). Indeed, cerebrospinal fluid from patients with NMOSD induces neutrophil chemotaxis and activation, resulting in astrocyte activation to a proinflammatory phenotype (26). G-CSF also plays a detrimental role in patients with NMOSD (27).

The JAK/STAT signaling pathway plays a critical role in activation and regulation of immune responses (28). Dysregulation of the JAK/STAT pathway is associated with many pathological conditions, including MS $(29,30)$. We previously demonstrated that inhibition of the JAK/STAT pathway ameliorates disease severity in a number of EAE models (31). STAT3 has been identified as an MS susceptibility gene (32, 33), and hyperactivation of STAT3 is observed in PBMCs from MS patients and correlates with disease progression (34). SOCS are a family of proteins that negatively regulate the JAK/STAT pathway (35). SOCS3 inhibits activation of STAT1 and STAT3, thereby terminating signaling through this pathway (36-38). Reduced SOCS3 expression has been observed in PBMCs from MS patients during relapses, which correlates with enhanced STAT3 activation (39). In NMOSD, clinical benefit has been observed with anti-IL-6R antibody treatment (40). The deleterious role of IL-6 is speculated to be mediated, in part, by IL-6 expansion of Th17 cells $(41,42)$, which occurs through a STAT3-dependent mechanism (43).

There are different phenotypes of EAE, including classical and atypical. Classical EAE is characterized by ascending paralysis with preferential immune cell infiltration in the spinal cord (5). Atypical EAE is associated with brain inflammation and characterized by ataxia and tremors. Some EAE models display a mixed phenotype of both classical and atypical clinical symptoms. We previously reported that deficiency of Socs 3 in myeloid cells (Socs $\left.3^{\mathrm{LLys}}\right)$ leads to a severe, nonresolving, atypical form of EAE $(44,45)$. Specifically, this brain-targeted, atypical EAE is dependent on preferential neutrophil infiltration in the cerebellum (44). However, the exact mechanism by which neutrophils induce atypical EAE is not clear. Here we demonstrate that neutrophils from Socs $3^{\Delta \mathrm{LysM}}$ mice produce excessive amounts of ROS, which is critical for the brain-targeted, atypical EAE phenotype. Furthermore, neutrophils lacking Socs3 are hypersensitive to G-CSF priming, which leads to enhanced oxidative burst in response to proinflammatory cytokines, and in vivo, G-CSF depletion significantly reduces atypical EAE disease progression. Overall, our findings demonstrate the importance of Socs 3 in controlling the hyperactivation of neutrophils and add to our understanding on the role of neutrophils in MS/EAE.

\section{Results}

Socs $3^{4 L y s M}$ mice exhibit brain-targeted EAE with infiltration of hyperactivated neutrophils. Socs ${ }^{3 \mathrm{LysM}}$ mice with atypical EAE exhibit nonresolving ataxia and tremor, along with preferential cerebellar neutrophil infiltration $(44,45)$. We assessed demyelination in this model using Black Gold staining, revealing that Socs $3^{\text {LysM }}$ mice exhibited cerebellar demyelination at the peak of EAE disease, whereas Socs $3^{\mathrm{t} / \mathrm{fl}}$ mice retained intact myelin structure in the cerebellum (Figure 1A).

Neutrophils have been implicated in both spinal cord-targeted, classical EAE $(19,20,46,47)$ as well as brain-targeted, atypical EAE (44, 48-50), although there is controversy about their exact functions. To address the role of neutrophils in atypical EAE, we first determined levels of surface receptors on cerebellar-infiltrating neutrophils using multicolor flow cytometry analysis (Supplemental Figure 1; supplemental material available online with this article; https://doi.org/10.1172/jci.insight.126520DS1). The cerebellar-infiltrating neutrophils in Socs $3^{\mathrm{ALys}}$ mice exhibited increased surface levels of CD $11 \mathrm{~b}$ and CXCR4 compared with Socs $3^{\mathrm{t} / \mathrm{fl}}$ mice, while surface levels of CD62L and CXCR2 were decreased in Socs ${ }^{\text {LysM }}$ mice compared with Socs $3^{\mathrm{fl} / \mathrm{fl}}$ mice (Figure 1, B and C). This altered surface marker expression profile has been associated with a primed neutrophil phenotype $(6,51-53)$. Primed neutrophils exhibit characteristics that include increased chemotaxis, increased degranulation, and enhanced production of ROS, and these phenotypes are observed in autoimmune conditions $(52,54)$. Consistent with a phenotype of increased chemotaxis, we found that the numbers of cerebellar neutrophils were significantly increased in Socs $3^{\mathrm{ALysM}}$ mice at the peak of EAE (Figure 1D). CD63 is a membrane protein associated with intracellular granules. During degranulation, CD63 is expressed on the cell surface as the granule 
A

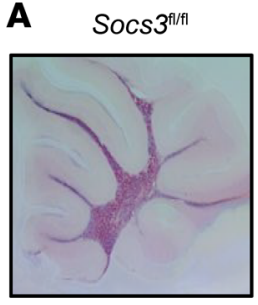

C

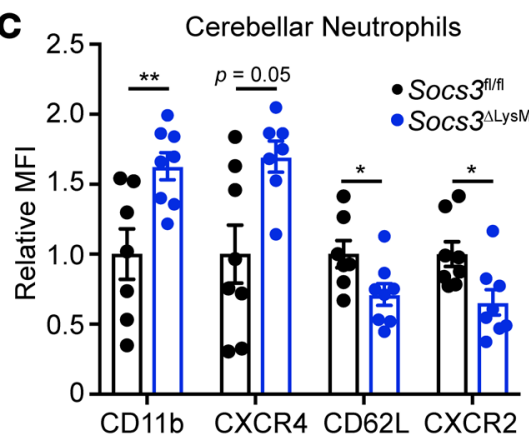

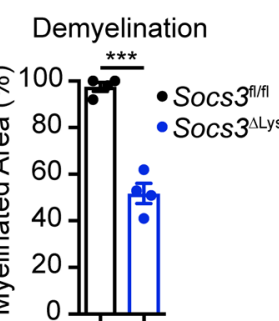

D
B $\quad$ D 11b

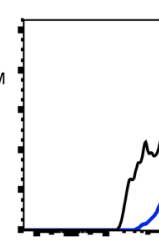

CXCR4

CD62L

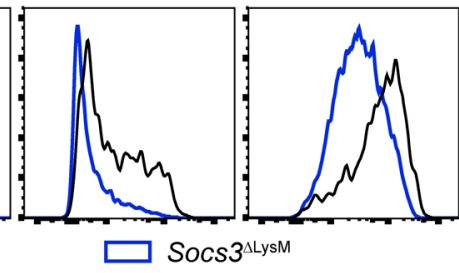
Neutrophils

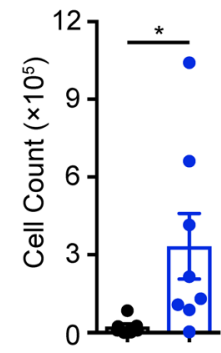

E Degranulation

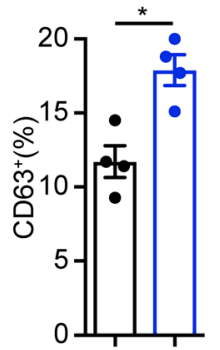

F $\mathrm{ROS}^{+}$

Neutrophils
I

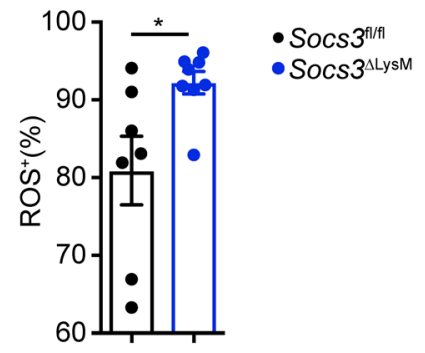

G

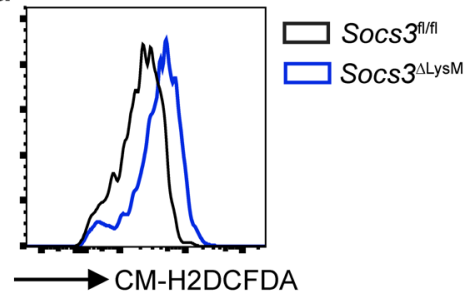

$J$

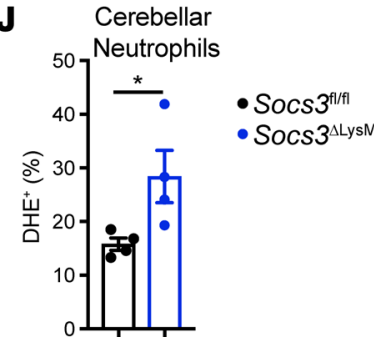

H ROS Production

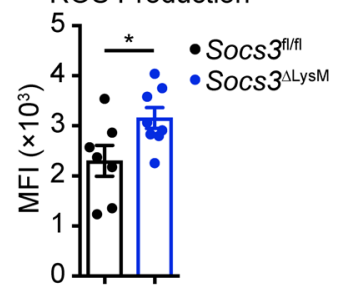

$\mathbf{K}$

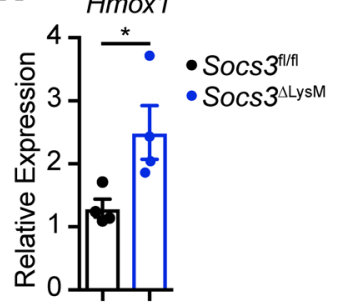

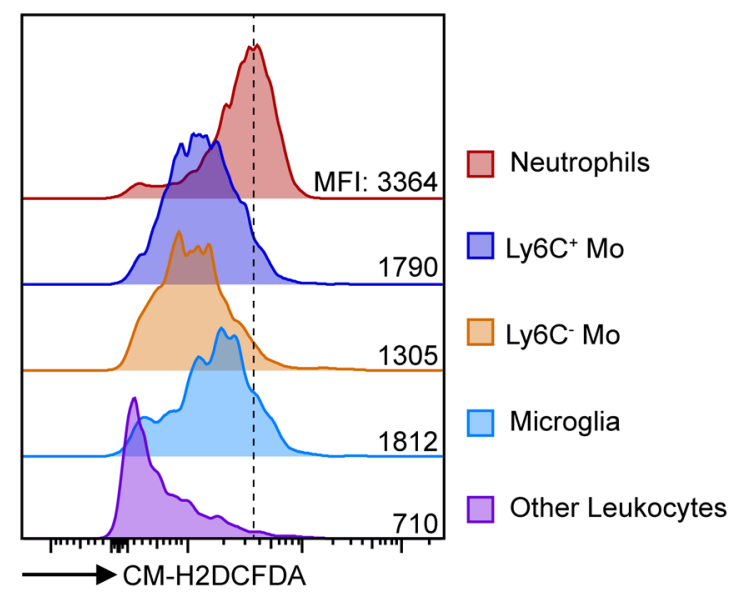

Figure 1. Socs $3^{\perp \mathrm{LysM}}$ mice exhibit brain-targeted, atypical EAE with infiltration of hyperactivated neutrophils. EAE was induced in both Socs $3^{\mathrm{fl} / \mathrm{fl}}$ and Socs $3^{\mathrm{ALys}}$ mice, and cerebellar tissue was collected at the peak of disease for further analysis. (A) Demyelination was assessed on day 14 and quantified by Black Gold staining $(n=4)$. Arrows indicate demyelinated regions. (B-J) Immune cells isolated from the cerebellum on days 13-14 using a Percoll gradient were subjected to surface staining. (B) Overlay of cerebellar-infiltrating neutrophils from Socs $3^{\mathrm{fl} / \mathrm{fl}}$ and Socs $3^{\Delta \mathrm{LysM}}$ mice stained for CD11b, CXCR4, CD62L, and CXCR2 ( $n=7-8)$. (C) Relative expression of surface markers of cerebellar-infiltrating neutrophils $(n=7-8)$. (D) Total number of cerebellar-infiltrating neutrophils ( $n=$ 7-8). (E) Degranulation was measured by analyzing the percentage of $C D 63^{+}$neutrophils on days $13-14(n=4)$. (F-I) Before surface staining, isolated neutrophils were incubated with CM-H2DCFDA $(1 \mu \mathrm{M})$ at $37^{\circ} \mathrm{C}$ for 30 minutes. (F) Percentage of ROS-producing neutrophils $(n=7-8)$. ( $(\mathbf{C}$ and $\mathbf{H})$ ROS production by neutrophils measured as the MFI of CM-H2DCFDA staining $(n=7-8)$. (I) ROS production by different immune cell types from the cerebellum on days $13-14$.

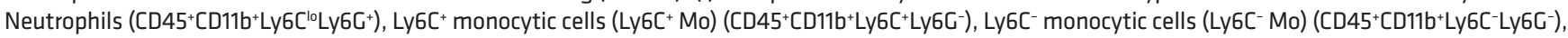
microglia (CD45 $\left.{ }^{\circ} \mathrm{CD} 11 \mathrm{~b}^{+}\right)$, and other leukocytes (CD45+CD11b-). Plot represents 8 individual samples. (J) Superoxide was measured using DHE $(n=4)$. (K) RNA was isolated from whole cerebellum on day 13 , and Hmox1 expression was analyzed by quantitative reverse transcription PCR (qRT-PCR) ( $n=4$ ). All error bars represent \pm SEM. ${ }^{*} P<0.05,{ }^{* *} P<0.01$, and ${ }^{* *} P<0.001$ by 2 -tailed Student's $t$ test. MFI, mean fluorescence intensity; DHE, dihydroethidium.

membrane and cell membrane merge (55). Analysis of surface expression of CD63 revealed that neutrophil degranulation was also increased in Socs $3^{\text {SLysM }}$ mice at the peak of EAE (Figure 1E).

We next assessed whether ROS production was altered in Socs $3^{\text {BLysM }}$ mice. To measure total ROS production, neutrophils isolated from the cerebellum were incubated with CM-H2DCFDA, a chemically reduced form of fluorescein used as an ROS indicator in live cells $(18,56)$. This revealed an increase in the level of ROS $^{+}$neutrophils in the cerebellum at the peak of EAE in Socs $3^{\text {ALys }}$ mice (Figure 1F). ROS production also increased on a per cell basis, as measured by an increase in the MFI of CM-H2DCFDA staining (Figure 1, G and $\mathrm{H}$ ). To determine whether neutrophils were the primary source of oxidative burst in 

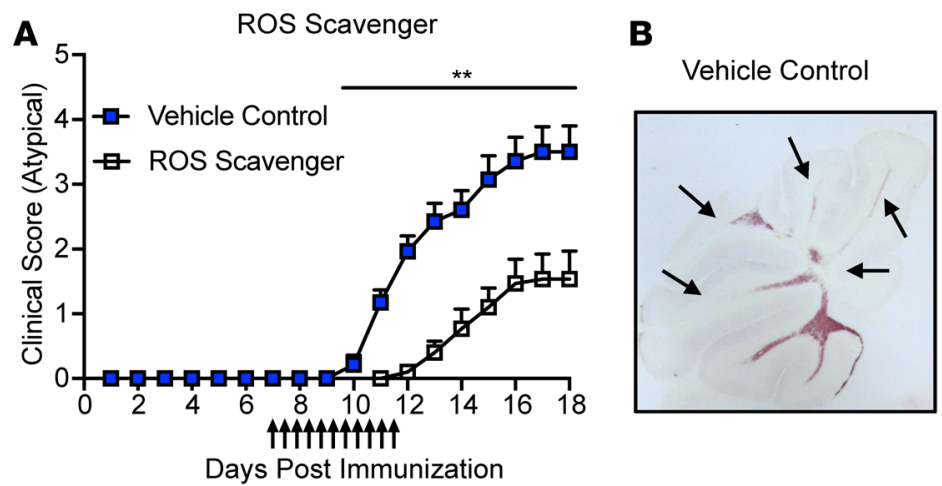

ROS Scavenger

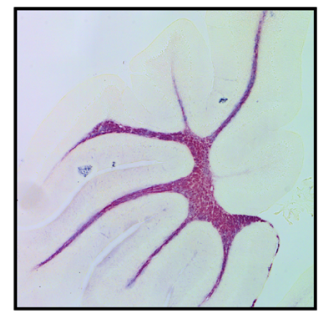

Demyelination

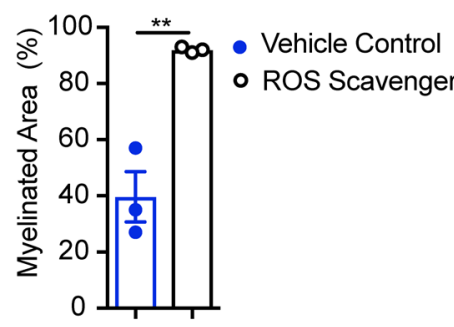

C

Cerebellum

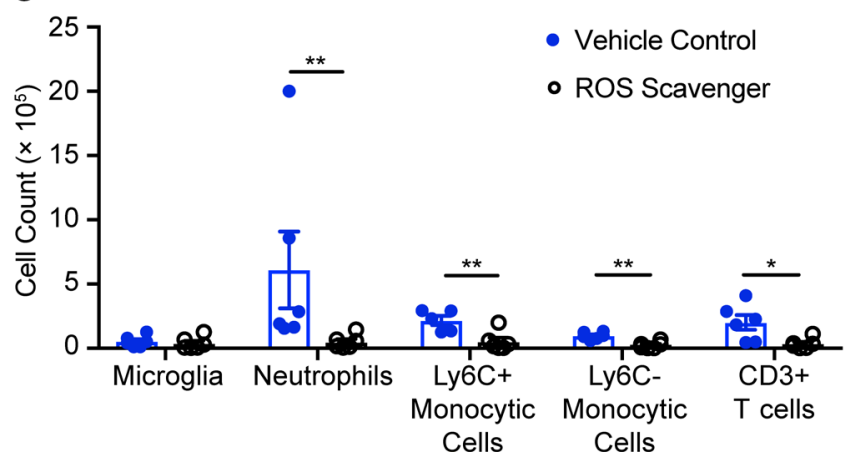

D $H \operatorname{mox} 1$

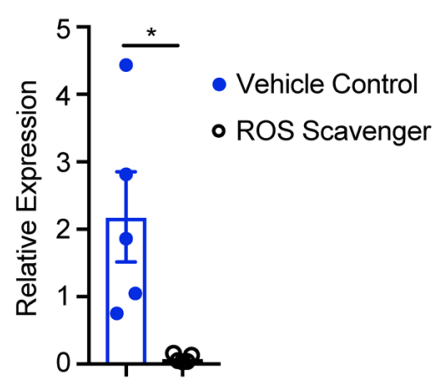

Figure 2. ROS plays an important role in the pathology of atypical EAE. EAE was induced in Socs $3^{\Delta L y s M}$ mice. Beginning on day 7 , ROS scavenger cocktail (FeTPPS $20 \mathrm{mg} / \mathrm{kg}$, PBN $50 \mathrm{mg} / \mathrm{kg}$, and EUK-134 $15 \mathrm{mg} / \mathrm{kg}$ ) was administered i.p. twice per day for 5 days. (A) Atypical EAE score of mice treated with ROS scavenger cocktail $(n=14)$ or vehicle control $(n=14)$. Mice that did not develop EAE (classical or atypical) were excluded. (B) Demyelination was assessed on day 14 and quantified by Black Gold staining $(n=3)$. Arrows indicate demyelinated regions. (C) On days 13 to 14 , immune cells from the cerebellum were

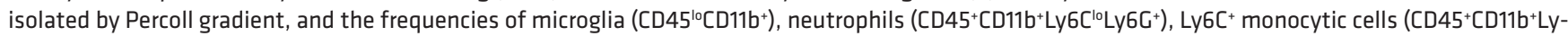

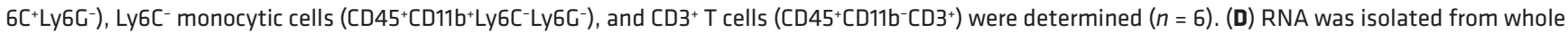
cerebellum on day 13 , and Hmox1 expression was analyzed by qRT-PCR $(n=5)$. All error bars represent \pm SEM. ${ }^{*} P<0.05$, and ${ }^{* *} P<0.01$ by Mann-Whitney rank-sum test (A) or 2-tailed Student's $t$ test (B-D).

the cerebellum of Socs $3^{\mathrm{L} y \mathrm{ys} M}$ mice, ROS production was measured in neutrophils, monocytic cells, microglia, and other leukocytes. As shown in Figure 1I, neutrophils produce the highest levels of ROS among these cell populations. Among all the species of free radicals, superoxide produced via NADPH oxidase 2 (NOX2) is considered harmful to endothelial cells and has detrimental roles in CNS inflammation (57-59). We measured superoxide by dihydroethidium (DHE) and consistent with total ROS levels, superoxide was also increased in cerebellar neutrophils from Socs $3^{\mathrm{L} \mathrm{ys} \mathrm{M}}$ mice (Figure $1 \mathrm{~J}$ ). To determine oxidative stress in Socs $3^{\Delta \mathrm{LysM}}$ mice, we measured the expression of Hmox1, a gene induced by ROS (60) in whole cerebellar tissue. Hmox 1 mRNA expression was increased at the peak of EAE in Socs $3^{\triangle \mathrm{LysM}}$ mice compared with Socs $3^{\mathrm{f} / \mathrm{fl}}$ mice (Figure $1 \mathrm{~K}$ ), indicating an enhanced oxidative stress. Together, these results demonstrate that brain-targeted, atypical EAE pathology in Socs $3^{\mathrm{LL} y s \mathrm{M}}$ mice is correlated with a primed neutrophil phenotype and enhanced ROS production.

ROS plays important roles in the pathology of brain-targeted $E A E$. We next determined whether oxidative stress contributes to the brain-targeted EAE phenotype. To neutralize ROS, we administered a cocktail

Table 1. ROS scavengers reduce the incidence of atypical EAE

\begin{tabular}{lcc}
\hline Treatment & Overall incidence & Classical $^{\mathbf{A}}$ \\
Vehicle control & $14 / 14(100 \%)$ & $7 / 14(50.0 \%)$ \\
ROS scavenger & $14 / 17(82.4 \%)$ & $12 / 17(70.5 \%)$
\end{tabular}

${ }^{A}$ Incidence was calculated from all mice. ${ }^{\mathrm{B}} P<0.01$ with $\chi^{2}$ test between vehicle control and ROS scavenger groups. 
of ROS scavengers to Socs $3^{\Delta \mathrm{LysM}}$ mice 7 days after immunization (56). Administration of ROS scavengers significantly delayed the time of onset and reduced the severity of brain-targeted EAE (Figure 2A). The incidence of brain-targeted EAE was also reduced (92.9\% to 47.1\%) (Table 1). Administration of ROS scavengers also prevented demyelination in the cerebellum (Figure 2B).

To better understand the underlying mechanism associated with the beneficial effect of ROS scavengers in atypical EAE, we assessed immune cell infiltration in mice treated with the ROS scavenger cocktail. On days 13 to 14 , we observed a significant reduction in neutrophils, monocytic cells, and $\mathrm{CD}^{+} \mathrm{T}$ cells infiltrating the cerebellum in mice treated with ROS scavengers; however, the frequency of microglia was comparable between vehicle control and ROS scavenger treatment (Figure 2C). Hmox 1 mRNA expression was significantly reduced in the cerebellar tissues from mice treated with ROS scavengers, indicating an overall reduction of oxidative stress (Figure 2D). To exclude the possibility that treatment with the ROS scavenger cocktail suppresses atypical EAE by affecting $T$ cell priming, we determined the frequency of myelin oligodendrocyte glycoprotein-specific (MOG-specific) T cells after treatment. The frequency of MOG-specific T cells, determined by cytokine production upon either MOG stimulation (Supplemental Figure 2A) or $\mathrm{MOG}_{38-49}$ tetramer staining (Supplemental Figure 2, C and D), were comparable between ROS scavenger treatment and vehicle control. The percentage of regulatory T cells after ROS scavenger treatment was also not changed (Supplemental Figure 2B).

Socs $3^{\mathrm{ALysM}}$ mice can exhibit classical EAE in addition to the predominant atypical EAE phenotype. There was not a significant change in the incidence or severity of classical EAE upon ROS scavenger treatment (Table 1 and Supplemental Figure 2E), indicating that the impact of ROS scavengers was specific to brain-targeted EAE. Collectively, these results demonstrate that ROS plays a critical role in the development of brain-targeted, atypical EAE but not in classical, spinal cord-targeted EAE.

G-CSF treatment promotes a primed phenotype in Socs3-deficient neutrophils via JAK1/STAT3. We next explored the mechanism underlying the primed neutrophil phenotype. SOCS3 negatively regulates STAT3 signaling and is induced upon activation of the JAK/STAT pathway (61). Therefore, we first determined which cytokines induce Socs3 expression in neutrophils. Activation of STAT3 by G-CSF in neutrophils has been well documented (37, 62-64); however, the literature is inconclusive about whether other cytokines, such as IL- 6 and IL-23, can activate neutrophils via STAT3 $(65,66)$. We found that G-CSF treatment induced strong Socs 3 expression 2 hours after stimulation and that this response was significantly higher than that induced by other cytokines, such as IL-6 and IL-23 (Figure 3A). In addition, G-CSF treatment led to enhanced STAT3 activation in Socs3-deficient neutrophils, in comparison to $\operatorname{Socs} 3^{\mathrm{f} / \mathrm{fl}}$ neutrophils (Figure 3, B and C). This difference was not observed in response to IL-6 (Figure 3, B and C) or IL-23 (Supplemental Figure 3, A and B). We previously reported that loss of Socs3 leads to persistent STAT3 activation in response to IFN- $\gamma$ stimulation in bone marrow-derived macrophages (36). However, in neutrophils, treatment with IFN- $\gamma$ induced only moderate expression of Socs 3 compared with treatment with G-CSF (Figure 3A), and IFN- $\gamma$ treatment did not lead to enhanced STAT3 activation in Socs3-deficient neutrophils in vitro (Supplemental Figure 3, C and D).

Upon G-CSF stimulation, Socs3-deficient neutrophils exhibited a primed phenotype in vitro, evident as increased surface expression of CD11b and CXCR4 and decreased surface expression of CD62L and CXCR2 relative to Socs $3^{\mathrm{fl} / \mathrm{fl}}$ neutrophils (Figure 3, D and E). In contrast, this difference for CD11b or CD62L was not observed upon stimulation with other cytokines, including IFN- $\gamma$, IL-17A/F, IL-6, IL-6 and sIL6R, IL-23, or IL-6 and IL-23 (Supplemental Figure 3E). We next asked whether the primed phenotype was due to activation of the JAK/STAT pathway. Pretreatment of Socs3-deficient neutrophils with AZD1480, a JAK1/2 inhibitor, or PF8041, a JAK1 inhibitor, impaired G-CSF-induced neutrophil priming (Figure $3 F$ ). These results indicate that G-CSF treatment activates the JAK1/STAT3 pathway to elicit a primed phenotype in Socs3-deficient neutrophils in vitro.

G-CSF induces a unique gene expression profile in Socs3-deficient neutrophils. We next determined whether G-CSF stimulation differentially affects gene expression in Socs3-deficient neutrophils. RNA-Seq analysis was performed on $\operatorname{Socs} 3^{\text {th/fl }}$ or Socs3-deficient neutrophils that were either unstimulated or stimulated with G-CSF. G-CSF stimulation affected the expression of a large number of genes in both Socs $3^{\mathrm{n} / \mathrm{fl}}$ and Socs3-deficient neutrophils, with more than 2,000 genes significantly upregulated or downregulated after stimulation (Figure 4A). Moreover, a total of 873 genes were upregulated, and 939 genes downregulated, specifically in Socs3-deficient neutrophils compared with Socs $3^{\text {fl/fl }}$ neutrophils (Figure 4B).

A gene set enrichment analysis (GSEA) revealed that a number of signaling pathways related to neutrophil activation were enhanced in Socs3-deficient neutrophils as compared with Socs $3^{\mathrm{n} / \mathrm{fl}}$ neutrophils 
A

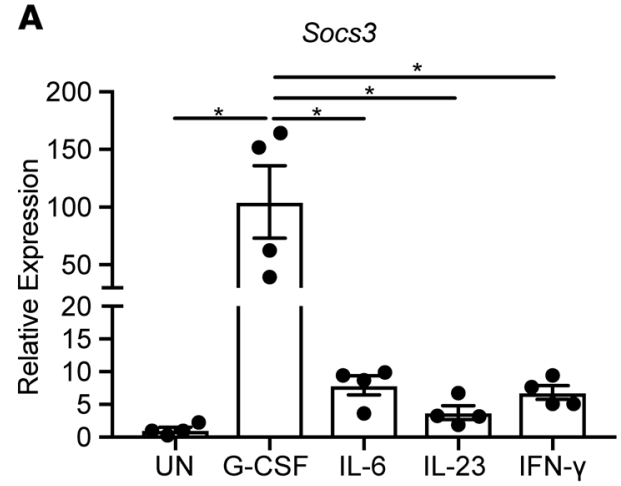

B

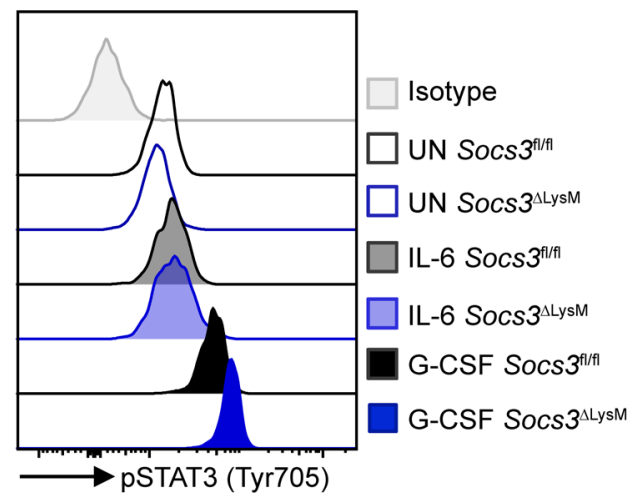

C $\quad$ PSTAT3 (Tyr705)

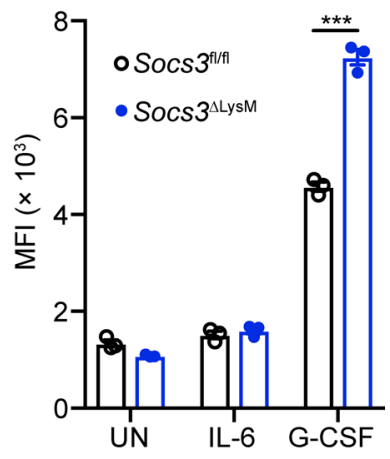

D
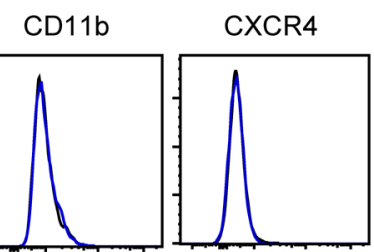

G-CSF
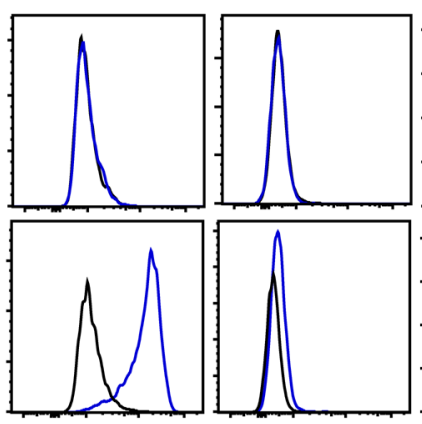

CD62L

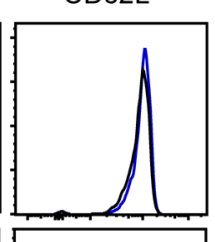

CXCR2

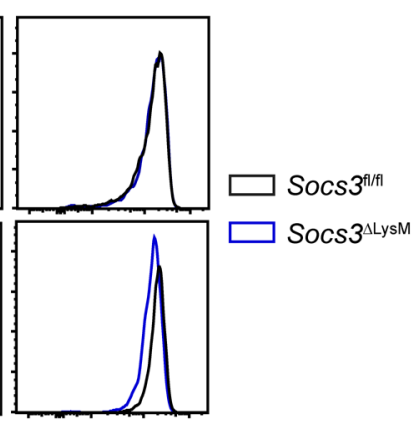

E

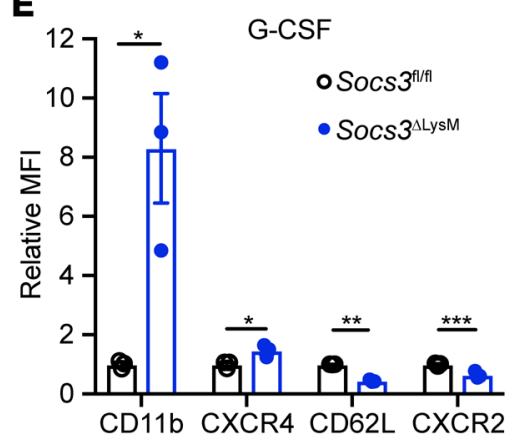

$\mathbf{F}$

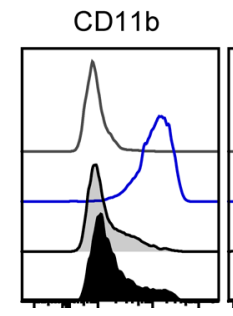

CXCR4

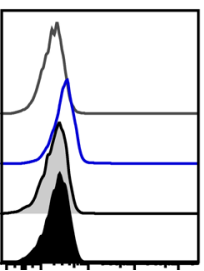

CD62L

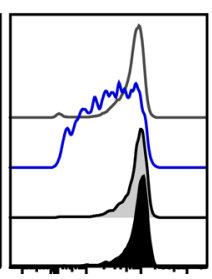

CXCR2

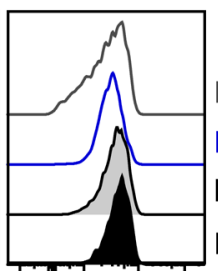

\section{$\square \mathrm{G}-\mathrm{CSF}$}

$\square$ G-CSF + AZD1480

G-CSF + PF8041

Figure 3. Socs3 deficiency promotes G-CSF hypersensitivity in neutrophils via JAK1 activation. (A) Bone marrow neutrophils were isolated from C57BL/6 mice and stimulated with G-CSF (10 ng/ml), IL-6 (100 ng/ml), IL-23 $(10 \mathrm{ng} / \mathrm{ml})$, or IFN- $\gamma(10 \mathrm{ng} / \mathrm{ml})$ for 2 hours. Expression of Socs3 mRNA was analyzed by qRT-PCR $(n=4)$. (B-E) Bone marrow neutrophils were isolated from Socs $3^{\mathrm{fl} / \mathrm{fl}}$ or Socs $3^{\Delta \mathrm{Lys} M}$ mice $(n=3)$. (B and C) Neutrophils were stimulated with G-CSF $(10 \mathrm{ng} / \mathrm{ml})$ or IL-6 $(100 \mathrm{ng} / \mathrm{ml})$ for 2 hours followed by intracellular staining for phosphorylated STAT3 (p-STAT3) (Y705). (D and E) Neutrophils were stimulated with G-CSF $(10 \mathrm{ng} / \mathrm{ml})$ for 8 hours, and expression of surface markers was analyzed. (F) Bone marrow neutrophils isolated from Socs $3^{\Delta \mathrm{LysM}}$ mice were pretreated with AZD1480 $(25 \mu \mathrm{M})$ or PF8041 $(25 \mu \mathrm{M})$ for 2 hours and then stimulated with G-CSF $(10 \mathrm{ng} / \mathrm{ml})$ for 8 hours, followed by surface staining. Plots

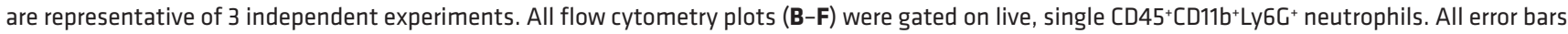
represent \pm SEM. ${ }^{*} P<0.05,{ }^{* *} P<0.01$, and ${ }^{* *} P<0.001$ by 1 -way ANOVA (A) or 2 -tailed Student's $t$ test (C and $\left.\mathbf{E}\right)$. MFI, mean fluorescence intensity.

upon G-CSF stimulation (Figure 4C). Upregulated pathways included the NF-kB pathway, which is related to neutrophil activation and chemotaxis in response to TNF- $\alpha$ and TLR ligands (67), and the STAT5 pathway, which is critical for neutrophils to respond to GM-CSF (68). Not surprisingly, STAT3 signaling was also upregulated in Socs3-deficient neutrophils upon G-CSF stimulation compared with Socs $3^{\text {t/f }}$ neutrophils. G-CSF mobilizes neutrophils by promoting chemotaxis, and our RNA-Seq data indicate that neutrophil migration-related genes were increased in Socs3-deficient neutrophils treated with G-CSF (Figure 4, C and D). Additionally, by qRT-PCR analysis, we confirmed that chemokines that promote neutrophil migration, including $\mathrm{Ccl} 4, \mathrm{Cxcl2}$, and $\mathrm{Cxcl3}$, were also enhanced in Socs3-deficient neutrophils compared with Socs $3^{\mathrm{A} / \mathrm{t}}$ neutrophils (Figure 4E). The RNA-Seq analysis also indicated that expression of genes related to the secretory vesicle trafficking pathway were increased in Socs3-deficient neutrophils, consistent with an increased potential for degranulation (Figure 4, C and D). In contrast, many of the downregulated pathways in Socs3-deficient neutrophils were associated with neutrophil resolution, including IFN- $\alpha$ responses (Figure 4C). DNA replication- and protein translation-related pathways were also reduced in Socs3-deficient neutrophils, indicating a maturation stage (Figure 4C). 
A

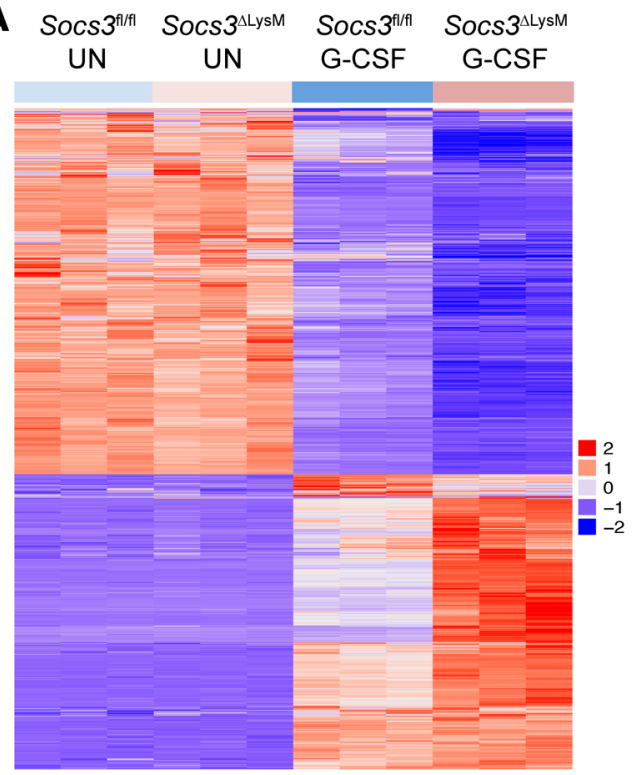

C

NF-kB Signaling
Response to Cytokines

STAT3 Signaling

MTORC1 Signaling

MYC Targets

Inflammatory Responses Innate Immune Responses

Leukocyte Migration

STAT5 Signaling Cell Activation

Toll Like Receptor Pathway

Cell Adhesion

p53 Pathway

TGF $\beta$ Pathway

Secretory Vesicles

Neutrophil Migration

Positive Regulation of ROS

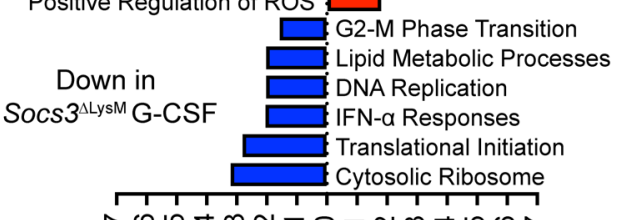

T

Normalized Enrichment Score (NES)
B

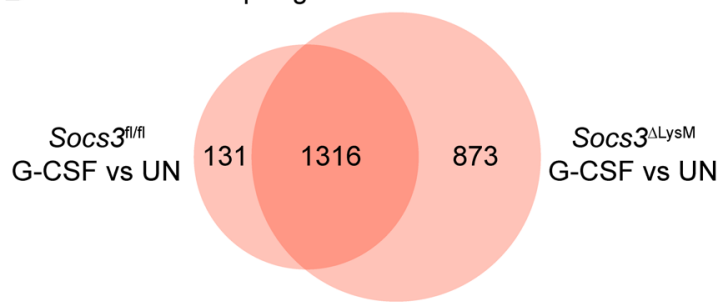

Down-regulated Genes

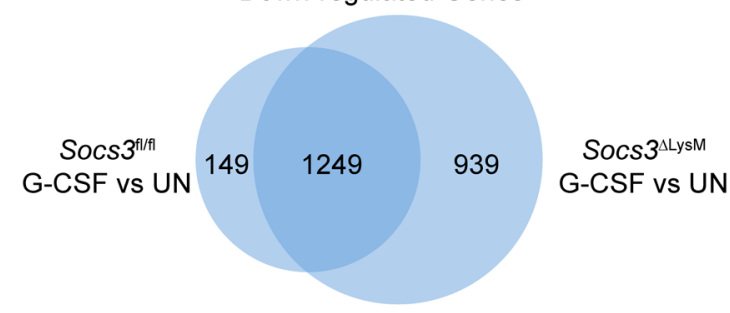

D

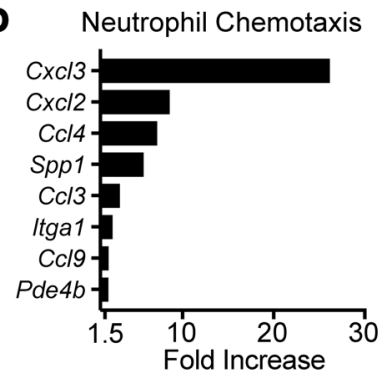

E

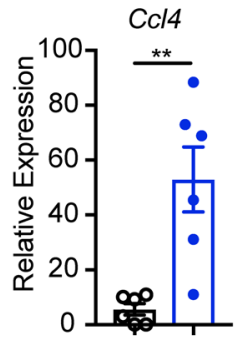

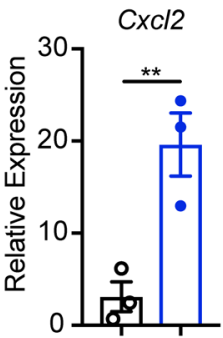

Secretory Vesicles

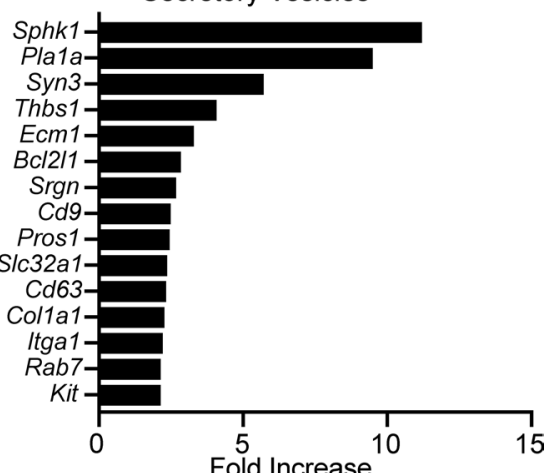

Figure 4. G-CSF induces a unique gene expression profile in Socs3-deficient neutrophils. Bone marrow neutrophils isolated from Socs $3^{\mathrm{fl} / \mathrm{fl}}$ and $\mathrm{Socs}^{\mathrm{ALys} \mathrm{M}}$ mice were stimulated with G-CSF (10 $\mathrm{ng} / \mathrm{ml})$ for 8 hours, followed by mRNA extraction and RNA-Seq ( $n=3$ ). (A) Heatmap of differentially expressed genes (DEGs) among the 4 groups. (B) Venn diagram depicting DEGs between Socs $3^{\mathrm{fl} / \mathrm{fl}}$ and Socs3-deficient neutrophils in response to G-CSF. (C) DEGs between Socs3-deficient neutrophils and Socs $3^{\mathrm{fl} / \mathrm{fl}}$ neutrophils upon G-CSF treatment were ranked based on the adjusted $P$ value and log fold change. GSEA illustrating upregulated and downregulated pathways in Socs3-deficient neutrophils in response to G-CSF. (D) Upregulated genes from selected pathways. (E) Bone marrow neutrophils isolated from Socs $3^{\mathrm{fl} / \mathrm{fl}}$ and Socs $3^{\mathrm{LLysM}}$ mice were stimulated with G-CSF (10 ng/ml) for 8 hours, followed by mRNA extraction and qRTPCR assay to determine mRNA expression of the indicated chemokines $(n=3-6)$. All error bars represent \pm SEM. ${ }^{* *} P<0.01$ by 2 -tailed Student's $t$ test.

To further determine whether G-CSF stimulated Socs3-deficient neutrophils are more activated compared with $\operatorname{Socs} 3^{\mathrm{fl} / \mathrm{fl}}$ neutrophils, we performed enrichment analysis using previously published RNA-Seq gene sets that contain genes expressed by activated neutrophils (stimulated with live bacteria, LPS, or TNF- $\alpha)(69,70$, 51). Genes that were increased by such activated neutrophils overlapped extensively with genes that were significantly upregulated in Socs3-deficient neutrophils upon G-CSF stimulation (Supplemental Figure 4). In addition, genes that were reduced after stimulation from the published data overlapped extensively with downregulated genes in Socs3-deficient neutrophils upon G-CSF stimulation (Supplemental Figure 4). Overall, the results from the RNA-Seq analysis indicate that upon G-CSF stimulation, Socs3-deficient neutrophils upregulate neutrophil activation genes and downregulate neutrophil resolution genes. 
A

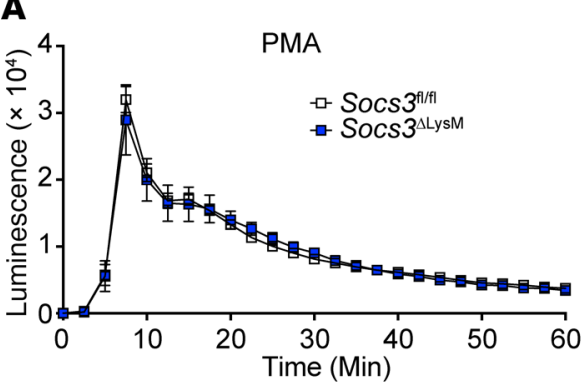

C

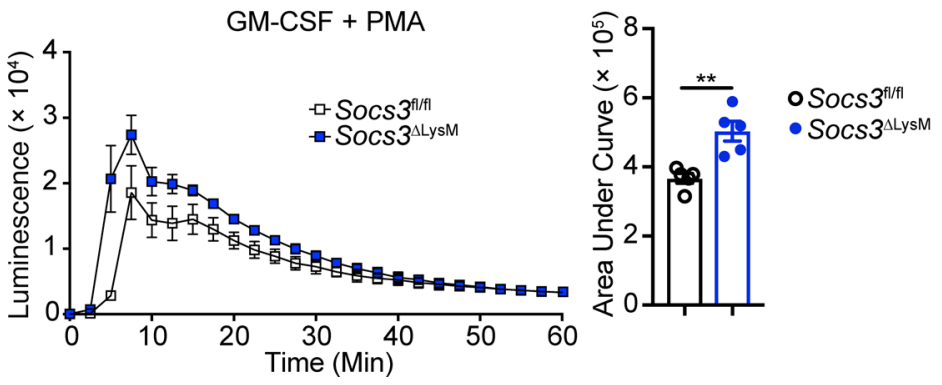

B

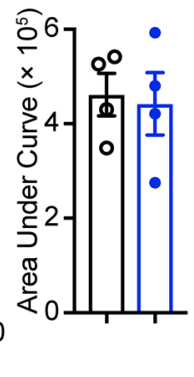

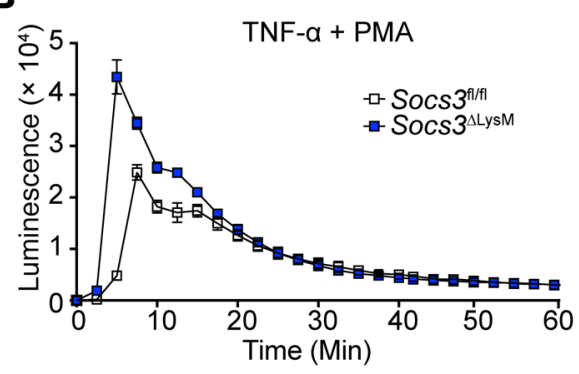

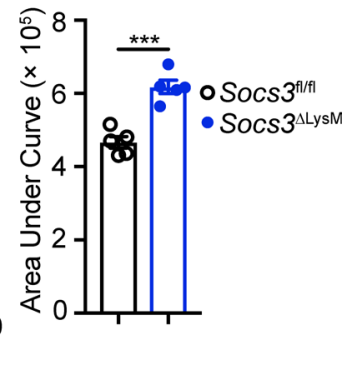

D LPS

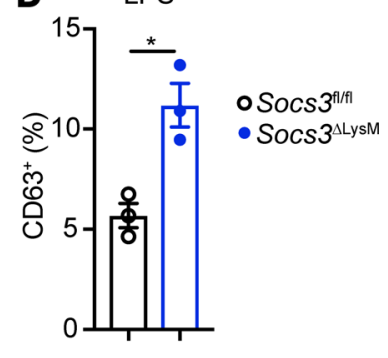

Figure 5. Socs3-deficient neutrophils are functionally hyperactivated in vitro upon G-CSF priming. Bone marrow neutrophils were isolated from Socs $3^{\mathrm{fl} / \mathrm{fl}}$ or Socs $3^{\mathrm{\Delta LysM}}$ mice and primed with G-CSF $(10 \mathrm{ng} / \mathrm{ml})$ for 12 hours. (A-C) Neutrophils were incubated with (A) PMA (10 ng/ml), (B) PMA plus TNF- $\alpha(10 \mathrm{ng} / \mathrm{ml})$, or (C) PMA plus GM-CSF $(10 \mathrm{ng} / \mathrm{ml})$, and production of ROS was measured by a luminol assay. Plot represents 4 to 5 independent experiments. (D) G-CSF-primed neutrophils were stimulated with LPS $(50 \mathrm{ng} / \mathrm{ml})$ for 1 hour, and degranulation was determined by surface staining of CD63 $(n=3)$. Flow cytometry plots were gated on live, single CD45+CD11b+Ly6C ${ }^{+}$neutrophils. All error bars represent \pm SEM. ${ }^{*} P<0.05,{ }^{* *} P<0.01$, and ${ }^{* * *} P<0.001$ by 2 -tailed Student's $t$ test $(\mathbf{B}-\mathbf{D})$.

Socs3-deficient neutrophils are functionally hyperactivated in vitro upon G-CSF priming. Based on the results in Figure 3 and the RNA-Seq data in Figure 4, we hypothesized that Socs3-deficient neutrophils are more sensitive to priming by G-CSF. To test this hypothesis, we first assessed ROS production by neutrophils using a luminol-based assay. For this assay, bone marrow neutrophils were pre-incubated with G-CSF overnight, followed by stimulation with PMA. In contrast with the expectation of our hypothesis, the data indicated that ROS production was comparable between Socs $3^{\mathrm{f} / \mathrm{fl}}$ and Socs3-deficient neutrophils (Figure 5A).

The RNA-Seq results indicated the possibility that the NF- $\mathrm{B}$ and STAT5 pathways were activated upon G-CSF priming of Socs3-deficient neutrophils (Figure 4C). Therefore, we next tested whether Socs3-deficient neutrophils were more sensitive to cytokines using the NF- $\kappa$ B and STAT5 pathways. TNF- $\alpha$ and GM-CSF are proinflammatory cytokines produced by $\mathrm{T}$ cells and myeloid cells in MS/EAE, and neutrophils are responsive to both cytokines $(67,68)$. Additionally, TNF- $\alpha-$ and GM-CSF-mediated induction of ROS plays a detrimental role during neuroinflammation by disrupting the blood-brain barrier (71, 72). To test the role of these cytokines in ROS induction, we treated neutrophils with TNF- $\alpha$ (Figure 5B) or GM-CSF (Figure 5C) after priming with G-CSF overnight. In the presence of PMA, both cytokines induced higher production of ROS in Socs3-deficient neutrophils compared with $\operatorname{Socs} 3^{\mathrm{n} / \mathrm{fl}}$ neutrophils. The G-CSF-primed, Socs3-deficient neutrophils also showed increased degranulation upon LPS stimulation compared with $\operatorname{Socs} 3^{\mathrm{f} / \mathrm{fl}}$ neutrophils (Figure 5D). Together, these results indicate that Socs3-deficient neutrophils become hyperactivated in vitro upon G-CSF priming.

G-CSF neutralization ameliorates brain-targeted EAE by preventing neutrophil inflitration and hyperactivation. Our results indicate that G-CSF leads to a primed phenotype in Socs3-deficient neutrophils in vitro and that this phenotype correlates with the incidence of brain-targeted EAE. Therefore, we next examined whether depleting G-CSF is beneficial for brain-targeted EAE. We first examined the levels of G-CSF during EAE, which revealed that G-CSF levels were lower in the plasma of Socs $3^{\mathrm{L} \text { LysM }}$ mice compared with Socs $3^{\mathrm{f} / \mathrm{fl}}$ mice before onset of EAE and were comparable throughout the onset stage and peak of EAE (Supplemental Figure 5A). The levels of G-CSF in the cerebellum were comparable between Socs $3^{3 \mathrm{LysM}}$ mice and Socs $3^{\mathrm{A} / \mathrm{fl}}$ mice (Supplemental Figure $5 \mathrm{~B}$ ). Next, we determined whether G-CSF depletion affected the development or phenotype of EAE. In previous studies, we found that neutrophils migrated into the spleen and lymph nodes throughout the course of EAE in both $\operatorname{Socs} 3^{\mathrm{f} / \mathrm{fl}}$ and $\operatorname{Socs} 3^{\mathrm{LLysM}}$ mice (data not shown). To avoid affecting the possible influence of neutrophils at the 

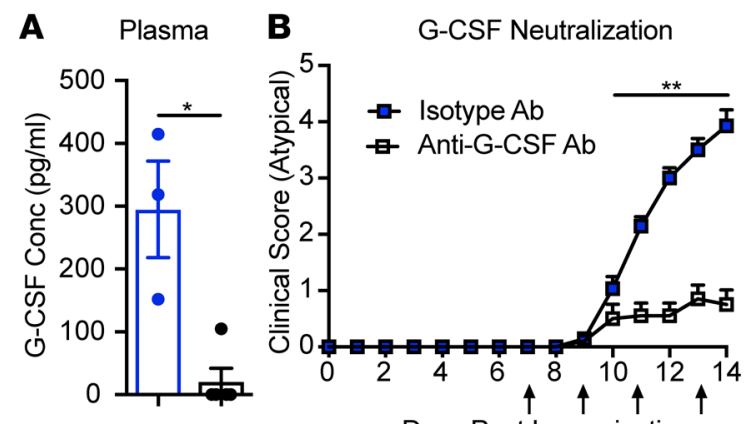

Days Post Immunization

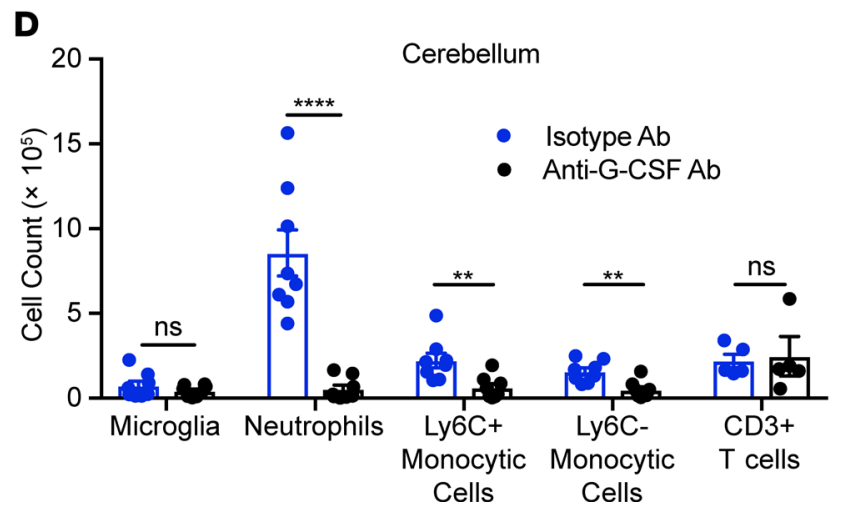

C Isotype Ab

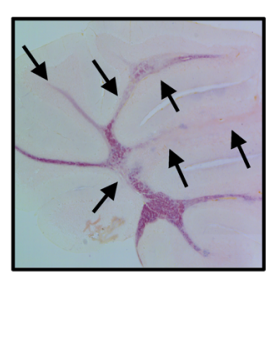

Anti-G-CSF Ab

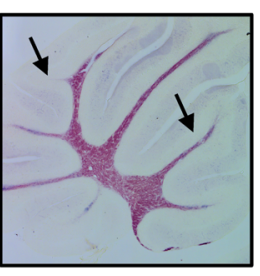

Demyelination

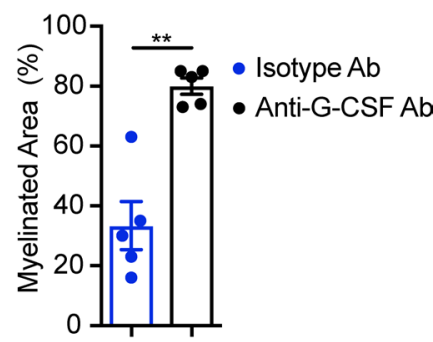

Figure 6. G-CSF neutralization suppresses brain-targeted, atypical EAE. EAE was induced in Socs $3^{\Delta \mathrm{LysM}}$ mice, and from day 7 , anti- $\mathrm{G}$-CSF Ab (20 $\mu$ g/ mouse) or isotype control Ab (20 $\mu \mathrm{g} /$ mouse) was administered i.p. every other day until day 13 . (A) On day 12 , plasma samples were collected and G-CSF levels determined by ELISA ( $n=3$ ). (B) Atypical EAE scoring of mice treated with anti-G-CSF Ab $(n=11)$ or isotype control Ab ( $n=14)$. Mice that did not develop EAE (classical or atypical) were excluded. (C) Demyelination was assessed and quantified by Black Gold staining ( $n=5$ ). Arrows indicate demyelinated regions. (D, E, and $\mathbf{G}$ ) Immune cells were isolated from the cerebellum 14 days after immunization ( $n=5-8)$. (D) Frequencies of microglia

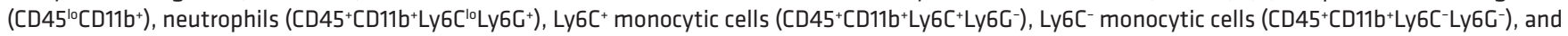
$\mathrm{CD3}^{+} \mathrm{T}$ cells $\left(\mathrm{CD} 45^{+} \mathrm{CD} 11 \mathrm{~b}^{-} \mathrm{CD}^{+}\right)$from the cerebella of mice treated with isotype Ab and anti-G-CSF Ab were determined. (E) Total numbers of ROS-producing neutrophils in the cerebella from mice treated with isotype Ab and anti-G-CSF Ab. (F) RNA was isolated from whole cerebellum, and Hmox1 expression was analyzed by qRT-PCR $(n=4)$. (G) Degranulation of cerebellar-infiltrating neutrophils from mice treated with isotype Ab or anti-G-CSF Ab was determined by surface expression of CD63. All error bars represent \pm SEM. ${ }^{*} P<0.05,{ }^{*} P<0.01,{ }^{* * *} P<0.0001$, and ns, not significant, by Mann-Whitney rank-sum test (B) or 2-tailed Student's $t$ test (A and $\mathbf{C}-\mathbf{G})$.

T cell priming stage, anti-G-CSF antibody $(\mathrm{Ab})$ was administered to mice beginning on day 7 after immunization. Administrating neutralizing Ab against G-CSF significantly reduced the levels of G-CSF in plasma (Figure 6A). Depletion of G-CSF significantly reduced the severity (Figure 6B) and incidence (Table 2) of brain-targeted EAE and reduced cerebellar demyelination (Figure 6C). A flow cytometry analysis indicated that G-CSF depletion significantly reduced the frequencies of infiltrating neutrophils and monocytic cells at the peak of EAE, while the frequencies of microglia and infiltrating $\mathrm{CD}^{+} \mathrm{T}$ cells were unchanged (Figure $6 \mathrm{D}$ ).

The reduced cerebellar infiltration (Figure 6D) correlated with a significant reduction in the frequency of neutrophils and monocytes that were present in the blood after G-CSF depletion (Supplemental Figure 6, A and B). In contrast, the frequency of $\mathrm{CD}^{+} \mathrm{T}$ cells present in the blood were not altered (Supplemental Figure 6B). Furthermore, the reduction of neutrophils in the blood did not affect neutrophil frequency in the spleen (Supplemental Figure 6C), suggesting a preferential reduction of CNS infiltration. Consistent with the significant reduction of infiltrating neutrophils in the cerebellum, the overall levels of oxidative stress were decreased, indicated by the reduction of ROS-producing neutrophils in the cerebellum (Figure 6E) and significantly reduced Hmox1 mRNA expression in the cerebellar tissues (Figure 6F). Neutrophil degranulation was also reduced in the cerebellum (Figure 6G). Interestingly, we did not observe a significant change in the incidence of classical EAE (Table 2) or disease severity (Supplemental Figure 7A). G-CSF neutralization did reduce infiltration of neutrophils in the spinal cord, whereas the frequency of microglia, monocytic cells, and $\mathrm{CD}^{+} \mathrm{T}$ cells was not affected (Supplemental Figure 7B). However, the change in neutrophil infiltration did not affect disease incidence or severity. Collectively, these results indicate that G-CSF neutralization ameliorates brain-targeted EAE. 
Table 2. Anti-G-CSF Ab reduces the incidence of atypical EAE

\begin{tabular}{lccc}
\hline Treatment & Overall incidence & Classical $^{\mathrm{A}}$ & Atypical $^{\mathrm{A}}$ \\
Isotype Ab & $14 / 14(100 \%)$ & $8 / 14(57.1 \%)$ & $14 / 14(100 \%)$ \\
Anti-G-CSF Ab & $11 / 14(78.6 \%)$ & $7 / 14(50.0 \%)$ & $5 / 14(35.7 \%)^{\mathrm{B}}$ \\
Ancidence was calculated from all mice. ${ }^{\mathrm{B}} P<0.01$ with $\chi^{2}$ test between anti-G-CSF Ab and isotype Ab groups. & \\
\hline
\end{tabular}

\section{Discussion}

We and others have demonstrated that brain-targeted, atypical EAE is predominantly a neutrophil-driven disease $(11,13,44,48,49,73,74)$. Previously, we reported that neutrophils increase production of proinflammatory cytokines, chemokines, and nitric oxide during onset of atypical EAE in Socs $3^{\text {LysM }}$ mice (44). In the present study, we have elucidated the cellular mechanisms by which neutrophils induce pathological changes in the cerebellum of Socs $3^{\mathrm{LLysM}}$ mice. We demonstrated that neutrophils from the cerebellum of Socs $3^{\mathrm{L} \mathrm{L} \mathrm{sM}}$ mice exhibit a hyperactivated phenotype and increase ROS production, which contributes to the development of atypical EAE. Mechanistically, we found that neutrophil hyperactivation is mediated by enhanced G-CSF signaling through the JAK/STAT pathway in Socs3-deficient neutrophils. Consistent with these results, we demonstrated that G-CSF neutralization has beneficial effects in brain-targeted, atypical EAE. These findings contribute to our understanding of the pathobiology of brain-targeted EAE and document the detrimental role of neutrophils in autoimmune neuroinflammation.

Recent studies suggest that neutrophils are quite heterogeneous and comprise different subsets with distinct functions $(6,75,76)$. Zhang et al. reported a unique subset of "aged" neutrophils characterized by high expression of CXCR4 and low expression of CD62L (51). These aged neutrophils represented an overly activated subset of neutrophils that were associated with increased proinflammatory activity and enhanced ROS production. Hawkins et al. reported that in a B cell-dependent, classical EAE model, CNS-infiltrating neutrophils specifically expressed the adhesion molecule ICAM1 (46). These ICAM1 ${ }^{+}$neutrophils exhibited increased surface expression of CD11b and CD45, formed synapses with T cells and B cells, and mediated inflammatory responses in vivo. These findings suggest that expression of specific neutrophil surface markers could be associated with distinct functions. In the current study, Socs3-deficient, hyperactivated neutrophils exhibited alterations in surface marker expression (elevated CD11b and CXCR4, decreased CD62L and CXCR2), enhanced ROS production, and increased degranulation both in vivo (Figure 1) and in vitro (Figure 3 and Figure 5). These results are consistent with clinical evidence showing that neutrophils from MS patients are characterized by increased TLR2 expression, elevated degranulation, resistance to apoptosis, and enhanced NET and ROS production, indicative of a hyperactivated status $(21,25)$.

The role of neutrophils in MS and EAE is complex. A number of studies, including from our group, indicate that neutrophils have detrimental functions in $\operatorname{EAE}(20,44,46-49,77)$. Neutrophil depletion significantly reduces the incidence and severity of atypical EAE (44). Furthermore, mice lacking G-CSF signaling are resistant to classical EAE, and neutrophil-related chemokine levels correlate with MS lesion burden (20). In addition, overexpression of CXCL1 in the CNS increased neutrophil infiltration and exacerbated demyelination in 2 mouse models of MS, and neutrophil ablation reduced the severity of demyelination, demonstrating a pathogenic role for neutrophils $(47,77)$. However, a recent study showed polymorphonuclear myeloid-derived suppressor cells (PMN-MDSCs) restrain accumulation of GM-CSF-producing B cells in the CNS, exerting a protective effect in classical EAE (78). Depletion of PMN-MDSCs after the onset of EAE led to activation of B cells in the CNS, subsequent microglial activation, and lack of recovery from the clinical signs of EAE. In addition, the number of neutrophils negatively correlates with that of B cells in the cerebrospinal fluid of MS patients (78). G-CSF administration at the onset of clinical symptoms reduces EAE severity in SJL/J mice by inhibiting recruitment of $\mathrm{T}$ cells to the CNS and suppressing TNF- $\alpha$ production (79). G-CSF administration on day 12 of classical EAE led to expansion of $\mathrm{Ly}_{6 \mathrm{G}}{ }^{+}$neutrophils and faster recovery from clinical symptoms than that of control-treated mice (78). In contrast, studies that show a detrimental role for neutrophils, including our findings, typically used models that impaired neutrophil function throughout the course of EAE or starting before the pre-onset phase $(20,44,48)$. Furthermore, there is complexity with respect to neutrophils and G-CSF, depending on the phenotype of EAE. Our findings are consistent with Pierson 
and Goverman (49), who demonstrated that neutrophils are not necessary for classical EAE. Regarding the role of G-CSF, Rumble et al. (20) demonstrated that mice lacking G-CSF signaling are resistant to classical EAE, using mice with global G-CSFR deletion. In our model, anti-G-CSF Ab was administered on day 7 after immunization and suppressed the brain-targeted, atypical EAE phenotype while not influencing classical EAE. These differences may reflect the timing at which G-CSF signaling is interrupted. Collectively, these findings indicate that the biological functions of neutrophils in EAE are timing, context, and model dependent, with both beneficial and detrimental behaviors (11-13, 15).

Our RNA-Seq analysis identified numerous genes that are differentially expressed in Socs3-deficient neutrophils in response to G-CSF, compared with $\operatorname{Socs} 3^{\text {fl/fl }}$ mice. Although some of these genes are likely direct targets of STAT3 based on GSEA, the majority are not. GSEA results implicate the NF- $\mathrm{kB}$ pathway as the most affected signaling pathway, with more than $150 \mathrm{NF}-\kappa \mathrm{B}$ target genes upregulated in Socs3-deficient neutrophils in response to G-CSF compared with Socs $3^{\mathrm{n} / \mathrm{fl}}$ mice. Previous studies have suggested that Socs3 negatively regulates the NF- $\mathrm{BB}$ pathway in macrophages (80), and our data suggest that Socs3 may also negatively regulate the NF- $\mathrm{KB}$ pathway in neutrophils. The RNA-Seq data also demonstrate that the STAT5 pathway, which GM-CSF activates, is enhanced in Socs3-deficient neutrophils after an 8-hour stimulation with G-CSF. G-CSF priming also rendered Socs3-deficient neutrophils more sensitive to GM-CSF treatment for ROS production. GM-CSF is an important cytokine implicated in EAE/MS (49, 72, 81-83). Our findings demonstrate that G-CSF priming of Socs3-deficient neutrophils, leading to hyperactivation of STAT3, render these cells more sensitive to GM-CSF stimulation. This may contribute, in part, to their detrimental functions. Another interesting finding from our RNA-Seq results is that neutrophils undergo metabolic changes in response to G-CSF, specifically increased glycolysis and reduced oxidative phosphorylation. Genes related to mTOR signaling and hypoxia were also upregulated after G-CSF treatment. Although the literature exploring the relationship between metabolism and neutrophil function is limited, activation of mTOR complex 1 and hypoxia-inducible factor- $1 \alpha$ have been shown to correlate with neutrophil activation and increased potential to form NETs (84-86). Previous studies have also demonstrated that Socs3-deficient neutrophils exhibit reduced apoptosis, consistent with our RNA-Seq results showing upregulation of antiapoptotic pathways $(37,38)$. Overall, our RNA-Seq analysis provides some potential downstream signaling pathways that may be involved in the hyperactivation of Socs3-deficient neutrophils and that will be characterized in the future.

Neutrophils are critically involved in the pathophysiology of numerous CNS diseases, including MS, NMOSD, Alzheimer's disease, and cerebral ischemia $(15,25,87-89)$. The findings from our study may have relevance to NMOSD in addition to MS, given the well-documented presence of neutrophils in CNS lesions and cerebrospinal fluid and findings that neutrophils contribute to astrocyte dysfunction and inflammation in NMOSD animal models $(25,26)$. Furthermore, neutrophils from patients with NMOSD exhibit some characteristics of activated neutrophils, including increased expression of TLR2 and $N$-formyl-methionyl-leucyl-phenylalanine (fMLP) receptors (25). Thus, the Socs $3^{\mathrm{ALys}}$ model with hyperactivated neutrophils may be of value in further studies of NMOSD pathophysiology. Finally, the composition of the gut microbiome has profound effects on MS and NMOSD pathogenesis (90-92). A role for the gut microbiome in regulating a disease-promoting, primed neutrophil phenotype has recently been described (51). It will be of interest to examine how modulation of the microbiota in Socs $3^{\Delta \mathrm{LysM}}$ mice may affect neutrophil function and subsequent neuroinflammatory responses in the CNS

\section{Methods}

Mice. C57BL/6 mice were bred in an animal facility at the University of Alabama at Birmingham (UAB). Transgenic mice with the Socs3 locus flanked with flox sequences (Socs $3^{\mathrm{f} / \mathrm{fl}}$; ref. 93) were the gift of Warren Alexander (Walter and Eliza Hall Institute of Medical Research, Victoria, Australia) and were bred at UAB. Socs3-conditional knockout (Socs $3^{\mathrm{ALys}}$ ) mice were generated by serial breeding of Socs $3^{\mathrm{t} / \mathrm{fl}}$ mice with mice expressing Cre recombinase under the control of the LysM promoter (45).

EAE induction and assessment. EAE was induced in Socs $3^{\mathrm{H} / \mathrm{fl}}$ mice and Socs $3^{\mathrm{LLysM}}$ mice by s.c. injection of MOG $_{35-55}$ emulsified in CFA (Hooke Laboratories, EK-2110), along with i.p. injection of 100 ng pertussis toxin (Hooke Laboratories, EK-2110) on day 0 and day 1, as previously described $(44,45,94)$. Mice experience a classical, atypical, or mixed phenotype after EAE induction. Classical EAE was scored as follows: 0 , no disease; 1 , decreased tail tone; 2 , hind limb weakness or partial paralysis; 3 , complete hind limb paralysis; 4 , front and hind limb paralysis; and 5, moribund state. Assessment of atypical EAE was as follows: 0, no disease; 1, hunched appearance, slight head tilt; 2 , ataxia, scruffy coat; 3 , severe head tilt, slight axial rotation, staggered walking; 
4, severe axial rotation, spinning; and 5, moribund. For the mixed phenotype, classical and atypical EAE were scored separately. For ROS scavenger treatment experiments, mice were administered either PBS containing 8\% DMSO (vehicle) or an ROS scavenger cocktail, which includes $20 \mathrm{mg} / \mathrm{kg}$ of FeTPPS (Cayman Chemical), 50 $\mathrm{mg} / \mathrm{kg}$ of phenyl $N$-t-butylnitrone (PBN, MilliporeSigma), and $15 \mathrm{mg} / \mathrm{kg}$ of EUK-134 (MilliporeSigma), by i.p. injection twice a day for 5 to 6 days (56). For G-CSF depletion, $20 \mu \mathrm{g}$ of anti-G-CSF Ab (R\&D Systems, MAB414) or isotype control rat IgG1 Ab (R\&D Systems, MAB005) was administered per mouse every other day by i.p. injection from days 7 to 12 after EAE induction (95). Mice were sacrificed on days 13-14 at the peak of EAE development for histology or flow cytometry analysis.

Assessment of demyelination. At the peak of EAE, mice were anesthetized and intracardially perfused with PBS, followed by $4 \%$ paraformaldehyde. The cerebellum was fixed with $4 \%$ PFA at $4^{\circ} \mathrm{C}$ overnight and then dehydrated with 30\% sucrose. Cryoprotected brains were embedded in OCT compound (Thermo Fisher Scientific) and cryosectioned to produce $40-\mu \mathrm{m}$ slices in the sagittal plane from the center. Sections were stained using the Black Gold II Myelin Staining Kit (MilliporeSigma, AG105) (96). Images of stained sections were acquired with Keyence Microscope BZ-X800. Total arbor vitae (white matter) area and the myelinated area (area of Black Gold staining) of the cerebellum were measured using the Hybrid Cell Count module provided by Keyence Microscope. The Percentage of Myelinated Area module was defined as myelinated area divided by total arbor vitae (white matter) area.

Abs, cytokines, and inhibitors. For flow cytometry-related experiments, Abs directed against murine CD11b (M1/70), CD45 (30-F11), Ly6C (HK1.4), Ly6G (1A8), CXCR2 (SA044G4), CD62L (MEL-14), CD63 (NVG-2), CD3 (17A2), CD4 (GK1.5), CD8 (53-6.7), CD44 (IM7), IFN- $\gamma$ (XMG1.2), and IL-17A (TC1118H10.1) were from BioLegend. Abs against GM-CSF (MP1-22E9) and Foxp3 (FJK-16S) were from eBioscience. Tetramer Ab against $\mathrm{MOG}_{38-49}$ (GWYRSPFSRVVH) was a gift from Laurie Harrington (UAB). Abs against CXCR4 (2B11) were purchased from BD Bioscience, and Ab directed against p-STAT3 (Tyr705) was purchased from Cell Signaling Technology. The LIVE/DEAD Fixable Aqua Stain kit was from Thermo Fisher Scientific (L34957). Murine IL-6, IFN- $\gamma$, G-CSF, sIL-6R, IL-23, TNF- $\alpha$, and GM-CSF were purchased from BioLegend. Neutralizing anti-G-CSF Ab was from R\&D Systems. AZD1480, a JAK1/2 inhibitor, was a gift from AstraZeneca R\&D and dissolved in DMSO as previously described (31). PF8041, a JAK1 inhibitor, was provided by Pfizer and dissolved in DMSO.

Neutrophil isolation. Bone marrow cells were isolated from the femurs of C57BL/6 Socs $3^{\mathrm{f} / \mathrm{fl}}$ or Socs $3^{\mathrm{ALys}}$ mice as previously described $(36,44)$. Red blood cells were lysed by incubation in ACK lysis buffer for 1 minute. $\mathrm{CD} 45^{+} \mathrm{CD} 11 \mathrm{~b}^{+} \mathrm{Ly} 6 \mathrm{G}^{+}$neutrophils were isolated by flow cytometry and used for qRT-PCR assays. For functional assays, neutrophils were isolated using the negative selection procedure from the EasySep Mouse Neutrophil Enrichment Kit (Stemcell Biotechnologies).

Quantitative real-time PCR and RNA-Seq. Neutrophils isolated by flow cytometry were stimulated as indicated. Total RNA was purified from cells using Trizol reagent extraction, and 500 ng to $2 \mu \mathrm{g}$ of RNA was sent to GENEWIZ for RNA-Seq and bioinformatics analysis. RNA-Seq results were submitted to the National Center for Biotechnology Information Gene Expression Omnibus database for archiving under accession number GSE122353. For generation of the heatmap, all significantly regulated genes were used. For other analysis, genes with an FDR less than 0.05 with more than a 1.5-fold change were considered DEGs. Genes with normalized count below 50 were removed from the list to ensure the quality of DEGs. Pathway analysis was performed using the GSEA program available from the Broad Institute. For qRT-PCR analysis, 500-1,000 ng of RNA was used as a template for cDNA synthesis. We performed qRT-PCR using TaqMan primers purchased from Thermo Fisher Scientific. The resulting data were analyzed using the comparative cycle threshold method to calculate relative RNA quantities (94).

Flow cytometry. For surface protein detection, cells were incubated with Fc Block (Bio X Cell, 2.4G2) for 15 minutes and washed, followed by incubation with viability dye and the indicated Abs directed against cell surface proteins. CM-H2DCFDA, a general oxidative stress indicator, was used to detect total ROS production $(18,56)$. Cells were incubated with $1 \mu \mathrm{M}$ CM-H2DCFDA (Thermo Fisher Scientific, C6827) at $37^{\circ} \mathrm{C}$ for 30 minutes, followed by staining of surface proteins as described above. To detect NOX2-mediated superoxide production, cells were incubated with DHE (Thermo Fisher Scientific, D11347) on ice for 30 minutes, and surface staining was performed simultaneously (97). Fluorescence levels were analyzed by flow cytometry within 6 hours of staining without fixation. For intracellular cytokine staining, cells were stimulated with PMA $(25 \mathrm{ng} / \mathrm{ml})$ and ionomycin $(1 \mu \mathrm{g} / \mathrm{ml})$ in the presence of GolgiStop (BD Biosciences) for 4 hours and were permeabilized using the Foxp3 Staining Buffer Kit 
(eBioscience) as previously described (98). For MOG tetramer staining, cells were stained with tetramer at $37^{\circ} \mathrm{C}$ for 60 minutes (99).

To analyze levels of p-STAT3, cells were stimulated with various cytokines for the indicated times, then fixed with BD Phosflow Lyse/Fix Buffer (BD Biosciences, 558049) immediately after stimulation at $37^{\circ} \mathrm{C}$ for 10 minutes. Cells were then permeabilized with prechilled BD Phosflow Perm Buffer III (BD Biosciences, 558050) on ice for 30 minutes, followed by staining for p-STAT3 for 1 hour. Stained cells were analyzed by flow cytometry immediately after staining.

For analysis of cells from the in vivo EAE experiments, mice were sacrificed and whole-body perfusion was performed. Mononuclear cells were isolated from the cerebellum using a 30\%:70\% Percoll (GE Healthcare, 17089101) gradient. Cell phenotypes were determined based on surface and intracellular staining patterns analyzed by flow cytometry as previously described $(31,45)$. All flow cytometry data were analyzed using FlowJo software (Tree Star Inc.).

Luminol assay. Bone marrow neutrophils were incubated with G-CSF (10 ng/ml) for 12 hours, transferred to 96 -well plates $\left(2-5 \times 10^{5}\right.$ cells per well), and washed with HBSS. Neutrophils were incubated with PMA (MilliporeSigma, P1585) (10 ng/ml), the cytokine of interest, luminol (MilliporeSigma, 123072) (200 nM), and HRP (MilliporeSigma, P8375) (1.6 units/ml). Luminescence was measured immediately thereafter and every 2 minutes for 60 minutes (100).

Statistics. Significant differences between 2 groups were analyzed by Student's $t$ test distribution. Oneway ANOVA was used to compare differences between more than 2 samples, and the Mann-Whitney ranksum test was used for EAE scores. $P$ values less than 0.05 were considered statistically significant. All error bars represent \pm SEM.

Study approval. All animal experiments were reviewed and approved by the Institutional Animal Care and Use Committee of UAB.

\section{Author contributions}

ZY, HQ, and ENB designed and analyzed the experiments. ZY, WY, SAG, KSL, LP, HW, and FC performed the experiments. ZY, HQ, and ENB wrote the manuscript. JSD helped design and interpret experiments related to ROS detection. ASW and WC assisted with RNA-Seq data analysis.

\section{Acknowledgments}

This work was supported by NIH grants R01NS057563 (to ENB), R01AI061061 (to ASW), and R01HL128502 (to JSD) and National Multiple Sclerosis Society grant RG-1606-24794 (to HQ). The Comprehensive Flow Cytometry Core at UAB is supported by NIH grants P30 AR048311 and P30 AI27667. We thank Hubert Tse (UAB) and lab members for their assistance with the luminol assay. We thank Laurie Harrington for the reagents and assistance with tetramer staining.

Address correspondence to: Hongwei Qin, Department of Cell, Developmental and Integrative Biology, University of Alabama at Birmingham, 1918 University Blvd., MCLM 390, Birmingham, Alabama 35294, USA. Phone: 205.934.2573; Email: hqin@uab.edu. Or to: Etty (Tika) Benveniste, Department of Cell, Developmental and Integrative Biology, University of Alabama at Birmingham, 510 20th Street South, 1220 Faculty Office Tower, Birmingham, Alabama 35294, USA. Phone: 205.934.7667; Email: tika@uab.edu.

\footnotetext{
1. Dendrou CA, Fugger L, Friese MA. Immunopathology of multiple sclerosis. Nat Rev Immunol. 2015;15(9):545-558.

2. Reich DS, Lucchinetti CF, Calabresi PA. Multiple sclerosis. NEngl J Med. 2018;378(2):169-180.

3. Thompson AJ, Baranzini SE, Geurts J, Hemmer B, Ciccarelli O. Multiple sclerosis. Lancet. 2018;391(10130):1622-1636.

4. Hemmer B, Kerschensteiner M, Korn T. Role of the innate and adaptive immune responses in the course of multiple sclerosis. Lancet Neurol. 2015;14(4):406-419.

5. Robinson AP, Harp CT, Noronha A, Miller SD. The experimental autoimmune encephalomyelitis (EAE) model of MS: utility for understanding disease pathophysiology and treatment. Handb Clin Neurol. 2014;122:173-189.

6. Nicolás-Ávila JÁ, Adrover JM, Hidalgo A. Neutrophils in Homeostasis, Immunity, and Cancer. Immunity. 2017;46(1):15-28.

7. Wang J, Hossain M, Thanabalasuriar A, Gunzer M, Meininger C, Kubes P. Visualizing the function and fate of neutrophils in sterile injury and repair. Science. 2017;358(6359):111-116.

8. Mayadas TN, Cullere X, Lowell CA. The multifaceted functions of neutrophils. Annu Rev Pathol. 2014;9:181-218.

9. Nauseef WM, Borregaard N. Neutrophils at work. Nat Immunol. 2014;15(7):602-611.

10. Soehnlein O, Steffens S, Hidalgo A, Weber C. Neutrophils as protagonists and targets in chronic inflammation. Nat Rev Immunol. 2017;17(4):248-261
} 
11. Pierson ER, Wagner CA, Goverman JM. The contribution of neutrophils to CNS autoimmunity. Clin Immunol. 2018;189:23-28.

12. Casserly CS, Nantes JC, Whittaker Hawkins RF, Vallières L. Neutrophil perversion in demyelinating autoimmune diseases: Mechanisms to medicine. Autoimmun Rev. 2017;16(3):294-307.

13. Miller NM, Wang J, Tan Y, Dittel BN. Anti-inflammatory mechanisms of IFN- $\gamma$ studied in experimental autoimmune encephalomyelitis reveal neutrophils as a potential target in multiple sclerosis. Front Neurosci. 2015;9:287.

14. Saadoun S, et al. Neutrophil protease inhibition reduces neuromyelitis optica-immunoglobulin G-induced damage in mouse brain. Ann Neurol. 2012;71(3):323-333.

15. Woodberry T, Bouffler SE, Wilson AS, Buckland RL, Brüstle A. The emerging role of neutrophil granulocytes in multiple sclerosis. J Clin Med. 2018;7(12):E511.

16. Lévesque SA, et al. Myeloid cell transmigration across the CNS vasculature triggers IL-1ß-driven neuroinflammation during autoimmune encephalomyelitis in mice. J Exp Med. 2016;213(6):929-949.

17. Jiang W, St-Pierre S, Roy P, Morley BJ, Hao J, Simard AR. Infiltration of CCR $2^{+}$Ly $6 C^{\text {high }}$ proinflammatory monocytes and neutrophils into the central nervous system is modulated by nicotinic acetylcholine receptors in a model of multiple sclerosis J Immunol. 2016;196(5):2095-2108.

18. Steinbach K, Piedavent M, Bauer S, Neumann JT, Friese MA. Neutrophils amplify autoimmune central nervous system infiltrates by maturing local APCs. J Immunol. 2013;191(9):4531-4539.

19. Aubé B, et al. Neutrophils mediate blood-spinal cord barrier disruption in demyelinating neuroinflammatory diseases. $J$ Immunol. 2014;193(5):2438-2454.

20. Rumble JM, et al. Neutrophil-related factors as biomarkers in EAE and MS. J Exp Med. 2015;212(1):23-35.

21. Naegele M, Tillack K, Reinhardt S, Schippling S, Martin R, Sospedra M. Neutrophils in multiple sclerosis are characterized by a primed phenotype. J Neuroimmunol. 2012;242(1-2):60-71.

22. Openshaw H, et al. Multiple sclerosis flares associated with recombinant granulocyte colony-stimulating factor. Neurology. 2000;54(11):2147-2150.

23. Rust H, Kuhle J, Kappos L, Derfuss T. Severe exacerbation of relapsing-remitting multiple sclerosis after G-CSF therapy. Neurol Neuroimmunol Neuroinflamm. 2016;3(2):e215.

24. Wingerchuk DM, et al. International consensus diagnostic criteria for neuromyelitis optica spectrum disorders. Neurology. 2015;85(2):177-189

25. Hertwig L, et al. Distinct functionality of neutrophils in multiple sclerosis and neuromyelitis optica. Mult Scler. 2016;22(2):160-173

26. Piatek P, et al. C5a-preactivated neutrophils are critical for autoimmune-induced astrocyte dysregulation in neuromyelitis optica spectrum disorder. Front Immunol. 2018;9:1694

27. Jacob A, et al. Detrimental role of granulocyte-colony stimulating factor in neuromyelitis optica: clinical case and histological evidence. Mult Scler. 2012;18(12):1801-1803

28. Villarino AV, Kanno Y, O'Shea JJ. Mechanisms and consequences of Jak-STAT signaling in the immune system. Nat Immunol. 2017;18(4):374-384

29. O'Shea JJ, Schwartz DM, Villarino AV, Gadina M, McInnes IB, Laurence A. The JAK-STAT pathway: impact on human disease and therapeutic intervention. Annu Rev Med. 2015;66:311-328.

30. Yan Z, Gibson SA, Buckley JA, Qin H, Benveniste EN. Role of the JAK/STAT signaling pathway in regulation of innate immunity in neuroinflammatory diseases. Clin Immunol. 2018;189:4-13.

31. Liu Y, et al. Therapeutic efficacy of suppressing the Jak/STAT pathway in multiple models of experimental autoimmune encephalomyelitis. J Immunol. 2014;192(1):59-72.

32. Cénit MC, et al. STAT3 locus in inflammatory bowel disease and multiple sclerosis susceptibility. Genes Immun. 2010;11(3):264-268

33. Jakkula E, et al. Genome-wide association study in a high-risk isolate for multiple sclerosis reveals associated variants in STAT3 gene. Am J Hum Genet. 2010;86(2):285-291

34. Frisullo G, et al. pSTAT1, pSTAT3, and T-bet expression in peripheral blood mononuclear cells from relapsing-remitting multiple sclerosis patients correlates with disease activity. J Neurosci Res. 2006;84(5):1027-1036.

35. Croker BA, Kiu H, Nicholson SE. SOCS regulation of the JAK/STAT signalling pathway. Semin Cell Dev Biol. 2008;19(4):414-422.

36. Qin H, Holdbrooks AT, Liu Y, Reynolds SL, Yanagisawa LL, Benveniste EN. SOCS3 deficiency promotes M1 macrophage polarization and inflammation. J Immunol. 2012;189(7):3439-3448.

37. Croker BA, et al. SOCS3 is a critical physiological negative regulator of G-CSF signaling and emergency granulopoiesis. Immunity. 2004;20(2):153-165.

38. Kimura A, et al. SOCS3 is a physiological negative regulator for granulopoiesis and granulocyte colony-stimulating factor receptor signaling. J Biol Chem. 2004;279(8):6905-6910.

39. Frisullo G, et al. The effect of disease activity on leptin, leptin receptor and suppressor of cytokine signalling- 3 expression in relapsing-remitting multiple sclerosis. J Neuroimmunol. 2007;192(1-2):174-183.

40. Araki M, et al. Efficacy of the anti-IL-6 receptor antibody tocilizumab in neuromyelitis optica: a pilot study. Neurology. 2014;82(15):1302-1306

41. Lin J, Li X, Xia J. Th17 cells in neuromyelitis optica spectrum disorder: a review. Int J Neurosci. 2016;126(12):1051-1060.

42. Barros PO, et al. Prediction of disease severity in neuromyelitis optica by the levels of interleukin (IL)-6 produced during remission phase. Clin Exp Immunol. 2016;183(3):480-489.

43. DuPage M, Bluestone JA. Harnessing the plasticity of CD4(+) T cells to treat immune-mediated disease. Nat Rev Immunol. 2016;16(3):149-163.

44. Liu Y, Holdbrooks AT, Meares GP, Buckley JA, Benveniste EN, Qin H. Preferential recruitment of neutrophils into the cerebellum and brainstem contributes to the atypical experimental autoimmune encephalomyelitis phenotype. J Immunol. 2015;195(3):841-852.

45. Qin H, et al. Signal transducer and activator of transcription-3/suppressor of cytokine signaling-3 (STAT3/SOCS3) axis in myeloid cells regulates neuroinflammation. Proc Natl Acad Sci USA. 2012;109(13):5004-5009.

46. Whittaker Hawkins RF, et al. ICAM1+ neutrophils promote chronic inflammation via ASPRV1 in B cell-dependent autoimmune encephalomyelitis. JCI Insight. 2017;2(23):e96882. 
47. Marro BS, Grist JJ, Lane TE. Inducible expression of CXCL1 within the central nervous system amplifies viral-induced demyelination. J Immunol. 2016;196(4):1855-1864.

48. Simmons SB, Liggitt D, Goverman JM. Cytokine-regulated neutrophil recruitment is required for brain but not spinal cord inflammation during experimental autoimmune encephalomyelitis. J Immunol. 2014;193(2):555-563.

49. Pierson ER, Goverman JM. GM-CSF is not essential for experimental autoimmune encephalomyelitis but promotes brain-targeted disease. JCI Insight. 2017;2(7):e92362.

50. Stoolman JS, et al. An IFN $\gamma /$ CXCL2 regulatory pathway determines lesion localization during EAE. J Neuroinflammation 2018;15(1):208

51. Zhang D, et al. Neutrophil ageing is regulated by the microbiome. Nature. 2015;525(7570):528-532.

52. Silvestre-Roig C, Hidalgo A, Soehnlein O. Neutrophil heterogeneity: implications for homeostasis and pathogenesis. Blood 2016;127(18):2173-2181.

53. Reber LL, et al. Neutrophil myeloperoxidase diminishes the toxic effects and mortality induced by lipopolysaccharide. $J$ Exp Med. 2017;214(5):1249-1258.

54. Wright HL, Moots RJ, Edwards SW. The multifactorial role of neutrophils in rheumatoid arthritis. Nat Rev Rheumatol. 2014;10(10):593-601

55. Martín-Martín B, Nabokina SM, Blasi J, Lazo PA, Mollinedo F. Involvement of SNAP-23 and syntaxin 6 in human neutrophil exocytosis. Blood. 2000;96(7):2574-2583

56. Noubade R, et al. NRROS negatively regulates reactive oxygen species during host defence and autoimmunity. Nature. 2014;509(7499):235-239.

57. Kim K, Li J, Tseng A, Andrews RK, Cho J. NOX2 is critical for heterotypic neutrophil-platelet interactions during vascular inflammation. Blood. 2015;126(16):1952-1964.

58. Park L, Anrather J, Girouard H, Zhou P, Iadecola C. Nox2-derived reactive oxygen species mediate neurovascular dysregulation in the aging mouse brain. J Cereb Blood Flow Metab. 2007;27(12):1908-1918.

59. Cooney SJ, Bermudez-Sabogal SL, Byrnes KR. Cellular and temporal expression of NADPH oxidase (NOX) isotypes after brain injury. J Neuroinflammation. 2013;10:155.

60. Broggi A, Tan Y, Granucci F, Zanoni I. IFN- $\lambda$ suppresses intestinal inflammation by non-translational regulation of neutrophil function. Nat Immunol. 2017;18(10):1084-1093.

61. Carow B, Rottenberg ME. SOCS3, a major regulator of infection and inflammation. Front Immunol. 2014;5:58.

62. Nguyen-Jackson H, Panopoulos AD, Zhang H, Li HS, Watowich SS. STAT3 controls the neutrophil migratory response to CXCR2 ligands by direct activation of G-CSF-induced CXCR2 expression and via modulation of CXCR2 signal transduction. Blood. 2010;115(16):3354-3363.

63. Zhang H, Nguyen-Jackson H, Panopoulos AD, Li HS, Murray PJ, Watowich SS. STAT3 controls myeloid progenitor growth during emergency granulopoiesis. Blood. 2010;116(14):2462-2471

64. Panopoulos AD, et al. STAT3 governs distinct pathways in emergency granulopoiesis and mature neutrophils. Blood. 2006;108(12):3682-3690.

65. Taylor PR, et al. Activation of neutrophils by autocrine IL-17A-IL-17RC interactions during fungal infection is regulated by IL-6, IL-23, ROR $\gamma$ t and dectin-2. Nat Immunol. 2014;15(2):143-151.

66. Wilkinson AN, et al. Granulocytes are unresponsive to IL-6 due to an absence of gp130. J Immunol. 2018;200(10):3547-3555.

67. McDonald PP, Bald A, Cassatella MA. Activation of the NF- $\mathrm{kB}$ pathway by inflammatory stimuli in human neutrophils. Blood. 1997;89(9):3421-3433

68. Kimura A, et al. The transcription factors STAT5A/B regulate GM-CSF-mediated granulopoiesis. Blood. 2009;114(21):4721-4728.

69. Schwartz JT, et al. Francisella tularensis alters human neutrophil gene expression: insights into the molecular basis of delayed neutrophil apoptosis. J Innate Immun. 2013;5(2):124-136.

70. Hutchins AP, Takahashi Y, Miranda-Saavedra D. Genomic analysis of LPS-stimulated myeloid cells identifies a common pro-inflammatory response but divergent IL-10 anti-inflammatory responses. Sci Rep. 2015;5:9100.

71. Sayed BA, Christy AL, Walker ME, Brown MA. Meningeal mast cells affect early T cell central nervous system infiltration and blood-brain barrier integrity through TNF: a role for neutrophil recruitment? J Immunol. 2010;184(12):6891-6900.

72. Spath S, et al. Dysregulation of the Cytokine GM-CSF induces spontaneous phagocyte invasion and immunopathology in the central nervous system. Immunity. 2017;46(2):245-260.

73. Quintana A, et al. Site-specific production of IL-6 in the central nervous system retargets and enhances the inflammatory response in experimental autoimmune encephalomyelitis. J Immunol. 2009;183(3):2079-2088.

74. Wensky AK, et al. IFN- $\gamma$ determines distinct clinical outcomes in autoimmune encephalomyelitis. J Immunol. 2005;174(3):1416-1423.

75. Takashima A, Yao Y. Neutrophil plasticity: acquisition of phenotype and functionality of antigen-presenting cell. J Leukoc Biol. 2015;98(4):489-496.

76. Evrard M, et al. Developmental analysis of bone marrow neutrophils reveals populations specialized in expansion, trafficking, and effector functions. Immunity. 2018;48(2):364-379.e8.

77. Grist JJ, et al. Induced CNS expression of CXCL1 augments neurologic disease in a murine model of multiple sclerosis via enhanced neutrophil recruitment. Eur J Immunol. 2018;48(7):1199-1210.

78. Knier B, et al. Myeloid-derived suppressor cells control B cell accumulation in the central nervous system during autoimmunity. Nat Immunol. 2018;19(12):1341-1351.

79. Zavala F, Abad S, Ezine S, Taupin V, Masson A, Bach JF. G-CSF therapy of ongoing experimental allergic encephalomyelitis via chemokine- and cytokine-based immune deviation. J Immunol. 2002;168(4):2011-2019.

80. Zhang X, et al. Macrophage/microglial Ezh2 facilitates autoimmune inflammation through inhibition of Socs3. J Exp Med. 2018;215(5):1365-1382.

81. Codarri L, et al. ROR $\gamma$ t drives production of the cytokine GM-CSF in helper T cells, which is essential for the effector phase of autoimmune neuroinflammation. Nat Immunol. 2011;12(6):560-567.

82. Croxford AL, Spath S, Becher B. GM-CSF in neuroinflammation: licensing myeloid cells for tissue damage. Trends Immunol. 2015;36(10):651-662. 
83. Duncker PC, Stoolman JS, Huber AK, Segal BM. GM-CSF promotes chronic disability in experimental autoimmune encephalomyelitis by altering the composition of central nervous system-infiltrating cells, but is dispensable for disease induction. J Immunol. 2018;200(3):966-973.

84. Weichhart T, Hengstschläger M, Linke M. Regulation of innate immune cell function by mTOR. Nat Rev Immunol. 2015;15(10):599-614.

85. Bao Y, et al. mTOR and differential activation of mitochondria orchestrate neutrophil chemotaxis. J Cell Biol. 2015;210(7):1153-1164

86. McInturff AM, et al. Mammalian target of rapamycin regulates neutrophil extracellular trap formation via induction of hypoxia-inducible factor $1 \alpha$. Blood. 2012;120(15):3118-3125.

87. Strecker JK, Schmidt A, Schäbitz WR, Minnerup J. Neutrophil granulocytes in cerebral ischemia - Evolution from killers to key players. Neurochem Int. 2017;107:117-126.

88. Cruz Hernández JC, et al. Neutrophil adhesion in brain capillaries reduces cortical blood flow and impairs memory function in Alzheimer's disease mouse models. Nat Neurosci. 2019;22(3):413-420.

89. Zenaro E, et al. Neutrophils promote Alzheimer's disease-like pathology and cognitive decline via LFA-1 integrin. Nat Med. 2015;21(8):880-886

90. Cignarella F, et al. Intermittent fasting confers protection in CNS autoimmunity by altering the gut microbiota. Cell Metab. 2018;27(6):1222-1235.e6.

91. Cree BA, Spencer CM, Varrin-Doyer M, Baranzini SE, Zamvil SS. Gut microbiome analysis in neuromyelitis optica reveals overabundance of Clostridium perfringens. Ann Neurol. 2016;80(3):443-447.

92. Cekanaviciute $\mathrm{E}$, et al. Gut bacteria from multiple sclerosis patients modulate human T cells and exacerbate symptoms in mouse models. Proc Natl Acad Sci U S A. 2017;114(40):10713-10718

93. Croker BA, et al. SOCS3 negatively regulates IL-6 signaling in vivo. Nat Immunol. 2003;4(6):540-545.

94. Gibson SA, et al. Protein Kinase CK2 controls the fate between Th17 cell and regulatory T cell differentiation. J Immunol. 2017;198(11):4244-4254.

95. Campbell IK, et al. Therapeutic targeting of the G-CSF receptor reduces neutrophil trafficking and joint inflammation in antibody-mediated inflammatory arthritis. J Immunol. 2016;197(11):4392-4402.

96. Liu L, et al. CXCR2-positive neutrophils are essential for cuprizone-induced demyelination: relevance to multiple sclerosis. Nat Neurosci. 2010;13(3):319-326.

97. Anderson JT, et al. Elevated levels of NO are localized to distal airways in asthma. Free Radic Biol Med. 2011;50(11):1679-1688.

98. Gibson SA, Yang W, Yan Z, Qin H, Benveniste EN. CK2 controls Th17 and regulatory T cell differentiation through inhibition of FoxO1. J Immunol. 2018;201(2):383-392.

99. Tian Y, Mollo SB, Harrington LE, Zajac AJ. IL-10 regulates memory T cell development and the balance between Th1 and follicular Th cell responses during an acute viral infection. J Immunol. 2016;197(4):1308-1321.

100. Padgett LE, Tse HM. NADPH oxidase-derived superoxide provides a third signal for CD4 T cell effector responses. J Immunol. 2016;197(5):1733-1742. 